\title{
On the stability of evolution Galerkin schemes applied to a two-dimensional wave equation system $^{1}$
}

\author{
M. Lukáčová-Medvidová, ${ }^{2}$ G. Warnecke $^{3}$ and Y. Zahaykah ${ }^{3}$
}

\begin{abstract}
The subject of the paper is the analysis of stability of the evolution Galerkin (EG) methods for the two-dimensional wave equation system. We apply von Neumann analysis and use the Fourier transformation to estimate the stability limits of both the first and the second order EG methods.
\end{abstract}

Key words: hyperbolic systems, wave equation, evolution Galerkin schemes, discrete Fourier transformation, amplification matrix, CFL condition.

\section{Introduction}

Evolution Galerkin methods (EG-methods) were proposed to approximate first order hyperbolic problems. These schemes were introduced by Morton et al., see, e.g., [8] for scalar problems and [9] for one-dimensional systems. The first generalization to two-dimensional systems was made in [23] by Ostkamp for the wave equation system as well for the Euler equations of gas dynamics. In [13] Lukáčová, Morton and Warnecke studied systematically approximate evolution operators and constructed new EG-schemes with better accuracy and stability properties. Further EG schemes as well as the approximate evolution operator of the solution for the wave equation system in three space dimensions were derived in [28]. These methods and their finite volume versions were applied to the linearized Euler equations and Maxwell equations [16]. Higher order finite volume EG-methods have been introduced and studied in [12], [14], [15] and [17]. In [11], [15], [6] the FVEG schemes have been generalized to fully nonlinear systems of hyperbolic conservation laws, such as the Euler equations of gas dynamics, shallow water equations as well as the shallow water magnetohydrodynamic equations. For hyperbolic conservation laws with source terms the so-called well-balanced FVEG schemes are proposed in [20]. In general, the FVEG schemes produce very accurate numerical solutions within the CPU time that is comparable to some other well-known finite volume methods. In particular, genuinely multidimensional features, such as oblique shocks, are resolved very well, cf., e.g. [11], [15]. For example, it has been shown in [15] that the global error of the second order FVEG scheme using (7.1)-(7.3) and (8.1)-(8.3) is approximately six times smaller than the error of the Lax-Wendroff (rotated Richtmyer) scheme as well as of the second order wave propagation algorithm of LeVeque [7].

The FVEG methods belong to the class of the so-called genuinely multidimensional schemes.

\footnotetext{
${ }^{1}$ This research was supported by the VolkswagenStiftung Agency, by the Deutsche Forschungsgemeinschaft Grant No. Wa 633/6-2 and partially by the European network HYKE, funded by the EC as contract HPRNCT-2002-00282. Authors gratefully acknowledge these supports.

${ }^{2}$ TU Hamburg-Harburg, Arbeitsbereich Mathematik, Schwarzenbergstrasse 95, 21073 Hamburg, Germany, email: lukacova@tu-harburg.de

${ }^{3}$ Institut für Analysis und Numerik, Otto-von-Guericke-Universität Magdeburg, Universitätsplatz 2, 39106 Magdeburg, Germany, emails: Gerald.Warnecke@mathematik.uni-magdeburg.de, Yousef.Zahaykah@mathematik.uni-magdeburg.de
} 
The goal is to have a method which approximates possibly all of the infinitely many directions of wave propagation. The reader is referred to [3], [4], [7], [22], [25], etc. for other genuinely multidimensional schemes.

The main objective of this paper is the analysis of the stability of the evolution Galerkin schemes. In [13] we have proven that the EG-schemes are conditionally stable. However, the precise stability limits have not been computed there. The goal of this paper is to find stability limits by analysis of the spectrum of the corresponding discrete operators for the EG as well as finite volume EG schemes, cf. [11], [13]. First, we consider the so-called EG3 scheme for the wave equation system in two-space dimension. We apply the discrete Fourier transform to obtain the amplification matrix. It turns out that its structure is too complex in order to derive precise stability limits theoretically. Anyway, we find theoretical stability estimates for a simplified problem. This is then compared with the experimental estimate of the spectrum of the amplification matrix of the EG3 scheme.

Further, we derive amplification matrices for the first- and the second-order finite volume schemes (FVEG) based on the approximate evolution operators. The spectral radius of the amplification matrices is estimated experimentally by a built-in Matlab procedure. Hence the stability limit of the schemes is estimated numerically.

The outline of this paper is as follows: in the next section we survey the general theory that we used to derive the exact integral equations. The exact integral equations as well as the approximate evolution operators for the two-dimensional wave equation system are given in Section 3. In Section 4 we recall the evolution Galerkin schemes. In Section 5 we introduce the discrete Fourier transform as well as the spectral norm that serve as tools in our analysis. In Section 6 we present the derivation of a stability condition for a simplified problem and compare the theoretical estimate, that we obtained by means of the Fourier analysis, with the experimental limit of the original problem. In Section 7 we consider the first-order finite volume schemes based on the approximate evolution operator $E_{\Delta}^{c o n s t}$. We determine the amplification matrices and estimate their stability limits. Finally in Section 8 we determine the amplification matrices of the second-order finite volume schemes based on the approximate evolution operator $E_{\Delta}^{b i l i n}$ and estimate the stability limits.

\section{General theory}

In this section we recall the exact integral equations for a general linear hyperbolic system using the concept of bicharacteristics. Consider a general form of linear hyperbolic system

$$
\mathbf{U}_{t}+\sum_{k=1}^{d} \mathcal{A}_{k} \mathbf{U}_{x_{k}}=0, \quad \mathbf{x}=\left(x_{1}, \ldots, x_{d}\right)^{T} \in \mathbb{R}^{d},
$$

where the coefficient matrices $\mathcal{A}_{k}, k=1, \ldots, d$, are elements of $\mathbb{R}^{p \times p}$ and the dependent variables are $\mathbf{U}=\left(u_{1}, \ldots, u_{p}\right)^{T}=\mathbf{U}(\mathbf{x}, t) \in \mathbb{R}^{p}$. Let $\mathcal{A}(\mathbf{n})=\sum_{k=1}^{d} n_{k} \mathcal{A}_{k}$ be the pencil matrix, where $\mathbf{n}=\left(n_{1}, \ldots, n_{d}\right)^{T}$ is a unit vector in $\mathbb{R}^{d}$. Since the system (2.1) is hyperbolic the matrix $\mathcal{A}(\mathbf{n})$ has $p$ real eigenvalues $\lambda_{k}, k=1, \ldots, p$, and $p$ corresponding linearly independent right eigenvectors $\mathbf{r}_{k}=\mathbf{r}_{k}(\mathbf{n}), k=1, \ldots, p$. Let $\mathcal{R}=\left[\mathbf{r}_{1}\left|\mathbf{r}_{2}\right| \ldots \mid \mathbf{r}_{p}\right]$ be the matrix of right eigenvectors. We define the characteristic variable $\mathbf{W}=\mathbf{W}(\mathbf{n})$ as $\partial \mathbf{W}(\mathbf{n})=\mathcal{R}^{-1} \partial \mathbf{U}$. Since the system (2.1) has constant coefficient matrices $\mathcal{A}_{k}$ we have $\mathbf{W}=\mathcal{R}^{-1} \mathbf{U}$ or $\mathbf{U}=\mathcal{R} \mathbf{W}$.

Transforming system (2.1) by multiplying it with $\mathcal{R}^{-1}$ from the left we get 


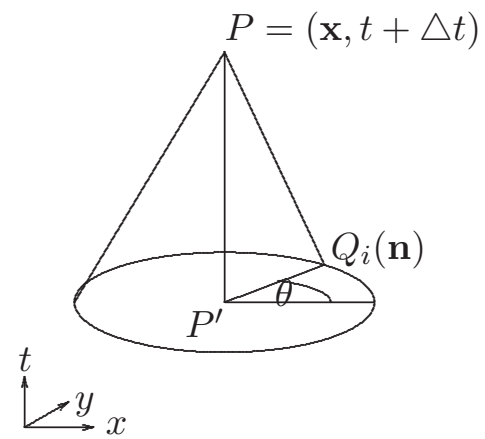

Figure 1: Bicharacteristics along the Mach cone through $P$ and $Q_{i}(\mathbf{n}), d=2$.

$$
\mathcal{R}^{-1} \mathbf{U}_{t}+\sum_{k=1}^{d} \mathcal{R}^{-1} \mathcal{A}_{k} \mathcal{R} \mathcal{R}^{-1} \mathbf{U}_{x_{k}}=0 .
$$

Let $\mathcal{B}_{k}=\mathcal{R}^{-1} \mathcal{A}_{k} \mathcal{R}=\left(b_{i j}^{k}\right)_{i, j=1}^{p}$, where $k=1,2, \ldots, d$, then the system $(2.2)$ can be rewritten in the following form using the characteristic variables

$$
\mathbf{W}_{t}+\sum_{k=1}^{d} \mathcal{B}_{k} \mathbf{W}_{x_{k}}=0
$$

Now we decompose $\mathcal{B}_{k}$ into the diagonal part $\mathcal{D}_{k}$ and the remaining part $\mathcal{B}_{k}^{\prime}$, i.e. $\mathcal{B}_{k}=\mathcal{D}_{k}+\mathcal{B}_{k}^{\prime}$. We obtain

$$
\mathbf{W}_{t}+\sum_{k=1}^{d} \mathcal{D}_{k} \mathbf{W}_{x_{k}}=-\sum_{k=1}^{d} \mathcal{B}_{k}^{\prime} \mathbf{W}_{x_{k}}=: \mathbf{S}
$$

The $i$-th bicharacteristic corresponding to the $i$-th equation of (2.3) is defined by

$$
\frac{d \mathbf{x}_{i}}{d \tilde{t}}=\mathbf{b}_{i i}(\mathbf{n})=\left(b_{i i}^{1}, b_{i i}^{2}, \ldots, b_{i i}^{d}\right)^{T},
$$

where $i=1, \ldots, p$. The diagonal entries $b_{i i}^{k}$ of the matrices $\mathcal{B}_{k}, k=1, \ldots, d, i=1, \ldots, p$, create the ray velocity vector $\mathbf{b}_{i i}$, cf. [1]. We consider the bicharacteristics backwards in time and set the initial conditions $\mathbf{x}_{i}(t+\Delta t, \mathbf{n})=\mathbf{x}$ for all $\mathbf{n} \in \mathbb{R}^{d}$ and $i=1, \ldots, p$, i.e. $\mathbf{x}_{i}(\tilde{t}, \mathbf{n})=\mathbf{x}-\mathbf{b}_{i i}(\mathbf{n})(t+\Delta t-\tilde{t})$.

We will integrate the $i$-th equation of the system (2.3) from the point $P \equiv(\mathbf{x}, t+\Delta t) \in \mathbb{R}^{p} \times \mathbb{R}_{+}$ down to the point $Q_{i}(\mathbf{n})=\left(\mathbf{x}_{i}(t, \mathbf{n}), t\right)=\left(\mathbf{x}-\Delta t \mathbf{b}_{i i}, t\right)$, where the bicharacteristic hits the plane at time $t$, see Figure 1. Note that bicharacteristics are straight lines because the system is linear and has constant coefficients. Now the $i$-th equation reads

$$
\frac{\partial w_{i}}{\partial t}+\sum_{k=1}^{d} b_{i i}^{k} \frac{\partial w_{i}}{\partial x_{k}}=-\left(\sum_{j=1, i \neq j}^{d}\left(b_{i j}^{1} \frac{\partial w_{j}}{\partial x_{1}}+b_{i j}^{2} \frac{\partial w_{j}}{\partial x_{2}}+\ldots+b_{i j}^{d} \frac{\partial w_{j}}{\partial x_{d}}\right)\right)=S_{i},
$$

Taking a vector $\sigma_{i}=\left(b_{i i}^{1}, b_{i i}^{2}, \ldots, b_{i i}^{d}, 1\right)$, we can define the directional derivative

$$
\frac{d w_{i}}{d \sigma_{i}}=\left(\frac{\partial w_{i}}{\partial x_{1}}, \frac{\partial w_{i}}{\partial x_{2}}, \ldots, \frac{\partial w_{i}}{\partial x_{d}}, \frac{\partial w_{i}}{\partial t}\right) \cdot \sigma_{i}=\frac{\partial w_{i}}{\partial t}+b_{i i}^{1} \frac{\partial w_{i}}{\partial x_{1}}+b_{i i}^{2} \frac{\partial w_{i}}{\partial x_{2}}+\ldots+b_{i i}^{d} \frac{\partial w_{i}}{\partial x_{d}} .
$$


Hence the $i$-th equation (2.4) can be rewritten as follows

$$
\frac{d w_{i}}{d \sigma_{i}}=S_{i}=-\sum_{j=1, i \neq j}^{d}\left(b_{i j}^{1} \frac{\partial w_{j}}{\partial x_{1}}+b_{i j}^{2} \frac{\partial w_{j}}{\partial x_{2}}+\ldots+b_{i j}^{d} \frac{\partial w_{j}}{\partial x_{d}}\right) .
$$

Integration from $P$ to $Q_{i}(\mathbf{n})$ gives

$$
w_{i}(P)-w_{i}\left(Q_{i}(\mathbf{n})\right)=S_{i}^{\prime}
$$

where

$$
S_{i}^{\prime}=\int_{t}^{t+\Delta t} S_{i}\left(\mathbf{x}_{i}(\tilde{t}, \mathbf{n}), \tilde{t}, \mathbf{n}\right) d \tilde{t}=\int_{0}^{\Delta t} S_{i}\left(\mathbf{x}_{i}(t+\Delta t-\tau, \mathbf{n}), t+\Delta t-\tau, \mathbf{n}\right) \mathrm{d} \tau .
$$

The reverse transformation of (2.5) into a system written in the original physical variables is done by multiplication with $\mathcal{R}$ from the left and $(d-1)$-dimensional integration of the variable $\mathbf{n}$ over the unit sphere $O$ in $\mathbb{R}^{d}$. This leads to the integral representation of the solution in the point $\mathbf{x}$ at time $t+\Delta t$ :

$$
\mathbf{U}(P)=\mathbf{U}(\mathbf{x}, t+\Delta t)=\frac{1}{|O|} \int_{O} \mathcal{R}(\mathbf{n})\left(\begin{array}{c}
w_{1}\left(Q_{1}(\mathbf{n}), \mathbf{n}\right) \\
w_{2}\left(Q_{2}(\mathbf{n}), \mathbf{n}\right) \\
w_{3}\left(Q_{3}(\mathbf{n}), \mathbf{n}\right) \\
\vdots \\
w_{p}\left(Q_{p}(\mathbf{n}), \mathbf{n}\right)
\end{array}\right) \mathrm{d} O+\tilde{\mathbf{S}}
$$

where

$$
\tilde{\mathbf{S}}=\left(\tilde{S}_{1}, \tilde{S}_{2}, \ldots, \tilde{S}_{p}\right)^{T}=\frac{1}{|O|} \int_{O} \mathcal{R}(\mathbf{n}) \mathbf{S}^{\prime} \mathrm{d} O=\frac{1}{|O|} \int_{O} \int_{0}^{\Delta t} \mathcal{R}(\mathbf{n}) \mathbf{S}(t+\Delta t-\tau, \mathbf{n}) \mathrm{d} \tau \mathrm{d} O
$$

and $|O|$ corresponds to the measure of the domain of integration.

\section{Exact integral equations and approximate evolution opera- tors for the wave equation system}

In this section we illustrate the application of the general theory of bicharacteristics for the two-dimensional system of wave equations. We recall the exact integral equations and present their possible approximation, the so-called EG3 approximate evolution operator. Consider the two-dimensional wave equation system

$$
\begin{aligned}
\phi_{t}+c\left(u_{x}+v_{y}\right) & =0, \\
u_{t}+c \phi_{x} & =0, \\
v_{t}+c \phi_{y} & =0,
\end{aligned}
$$

where $c$ is a given positive constant representing the speed of sound. We will recall here the exact integral equations derived in [13]. Let $P=(x, y, t+\Delta t), P^{\prime}=(x, y, t), Q=$ $(x+c \Delta t \cos \theta, y+c \Delta t \sin \theta, t)=(\mathbf{x}+c \Delta t \mathbf{n}(\theta), t)$ and let the so-called source term be given as

$$
S=c\left[u_{x} \sin ^{2} \theta-\left(u_{y}+v_{x}\right) \sin \theta \cos \theta+v_{y} \cos ^{2} \theta\right]
$$


then exact integral equations for the wave equation system (3.1) are given as

$$
\begin{aligned}
& \phi_{P}=\frac{1}{2 \pi} \int_{0}^{2 \pi}\left(\phi_{Q}-u_{Q} \cos \theta-v_{Q} \sin \theta\right) d \theta+\tilde{S}_{1} \\
& u_{P}=\frac{1}{2} u_{P^{\prime}}+\frac{1}{2 \pi} \int_{0}^{2 \pi}\left(-\phi_{Q} \cos \theta+u_{Q} \cos ^{2} \theta+v_{Q} \sin \theta \cos \theta\right) d \theta+\tilde{S}_{2} \\
& v_{P}=\frac{1}{2} v_{P^{\prime}}+\frac{1}{2 \pi} \int_{0}^{2 \pi}\left(-\phi_{Q} \sin \theta+u_{Q} \cos \theta \sin \theta+v_{Q} \sin ^{2} \theta d \theta+\tilde{S}_{3}\right.
\end{aligned}
$$

where

$$
\begin{aligned}
\tilde{S}_{1}= & \frac{-1}{2 \pi} \int_{0}^{2 \pi} \int_{0}^{\Delta t} S(\mathbf{x}+c \tau \mathbf{n}(\theta), t+\Delta t-\tau, \theta) \mathrm{d} \tau \mathrm{d} \theta \\
\tilde{S}_{2}= & \frac{1}{2 \pi} \int_{0}^{2 \pi} \int_{0}^{\Delta t} \cos \theta S(\mathbf{x}+c \tau \mathbf{n}(\theta), t+\Delta t-\tau, \theta) \mathrm{d} \tau \mathrm{d} \theta \\
& -\frac{1}{2 \pi} \int_{0}^{2 \pi} \int_{0}^{\Delta t}\left[c \phi_{x}(\mathbf{x}, t+\Delta t-\tau) \sin ^{2} \theta-c \phi_{y}(\mathbf{x}, t+\Delta t-\tau) \sin \theta \cos \theta\right] \mathrm{d} \tau \mathrm{d} \theta, \\
\tilde{S}_{3}= & \frac{1}{2 \pi} \int_{0}^{2 \pi} \int_{0}^{\Delta t} \sin \theta S(\mathbf{x}+c \tau \mathbf{n}(\theta), t+\Delta t-\tau, \theta) \mathrm{d} \tau \mathrm{d} \theta \\
& -\frac{1}{2 \pi} \int_{0}^{2 \pi} \int_{0}^{\Delta t}\left[c \phi_{y}(\mathbf{x}, t+\Delta t-\tau) \cos ^{2} \theta-c \phi_{x}(\mathbf{x}, t+\Delta t-\tau) \sin \theta \cos \theta\right] \mathrm{d} \tau \mathrm{d} \theta .
\end{aligned}
$$

The above integral equations give us an implicit formulation of the solution at the point $P=\left(x, y, t^{n+1}\right)$. In order to obtain an explicit numerical scheme it is necessary to use numerical quadrature rules in order to approximate the time integral from 0 to $\Delta t$. Using the backward rectangle rule leads to an $\mathcal{O}\left(\Delta t^{2}\right)$ approximation of the time integrals appearing in $\tilde{S}_{1}, \tilde{S}_{2}$ and $\tilde{S}_{3}$. Further we use the following result [13, Lemma 2.1]:

$$
\begin{aligned}
\Delta t \int_{0}^{2 \pi} S(t, \theta) \mathrm{d} \theta & =\int_{0}^{2 \pi}(u \cos \theta+v \sin \theta) \mathrm{d} \theta, \\
\Delta t \int_{0}^{2 \pi} S(t, \theta) \cos \theta \mathrm{d} \theta & =\int_{0}^{2 \pi}(u \cos 2 \theta+v \sin 2 \theta) \mathrm{d} \theta, \\
\Delta t \int_{0}^{2 \pi} S(t, \theta) \sin \theta \mathrm{d} \theta & =\int_{0}^{2 \pi}(u \sin 2 \theta+v \cos 2 \theta) \mathrm{d} \theta .
\end{aligned}
$$

Note that these formulae allow to replace the derivatives of our dependent variables in $S$ by the variables themselves. Rectangle rule approximation for the time integral and (3.6) yield 
the so-called EG3 approximate evolution operator:

\section{Approximate evolution operator for EG3}

$$
\begin{gathered}
\phi_{P}=\frac{1}{2 \pi} \int_{0}^{2 \pi}\left(\phi_{Q}-2 u_{Q} \cos \theta-2 v_{Q} \sin \theta\right) \mathrm{d} \theta+O\left(\Delta t^{2}\right), \\
u_{P}=\frac{1}{2} u_{P^{\prime}}+\frac{1}{2 \pi} \int_{0}^{2 \pi}\left(-2 \phi_{Q} \cos \theta+u_{Q}\left(3 \cos ^{2} \theta-1\right)+3 v_{Q} \sin \theta \cos \theta\right) \mathrm{d} \theta+O\left(\Delta t^{2}\right), \\
v_{P}=\frac{1}{2} v_{P^{\prime}}+\frac{1}{2 \pi} \int_{0}^{2 \pi}\left(-2 \phi_{Q} \sin \theta+3 u_{Q} \sin \theta \cos \theta+v_{Q}\left(3 \sin ^{2} \theta-1\right)\right) \mathrm{d} \theta+O\left(\Delta t^{2}\right) .
\end{gathered}
$$

We refer the reader to [13], [28] for other approximate evolution operators EG1, EG2, EG4. In what follows we will concentrate on the stability analysis of the EG3 scheme, for which the best numerical results have been obtained, see [13]. The stability analysis for other schemes can be done in an analogous way.

\section{Evolution Galerkin Schemes}

In this section we describe the evolution Galerkin schemes in the finite difference framework as well as the finite volume evolution Galerkin schemes. The main idea behind evolution Galerkin schemes is the following. Transported quantities are evolved in time along the bicharacteristics and then projected onto a finite element space. These methods connect finite element methods with the theory of bicharacteristics. In the finite volume framework the approximate operators are used only in order to compute fluxes on cell interfaces. Thus, instead of one-dimensional Riemann solvers, which work only in the normal directions to the cell interfaces, we compute the approximate solution at cell interfaces by a multi-dimensional evolution operator. This can be considered as a predictor step. In the corrector step the finite volume update is made.

Consider a mesh in $\mathbb{R}^{2}$, which consists of the square mesh cells

$$
\Omega_{k l}=\left[\left(k-\frac{1}{2}\right) h,\left(k+\frac{1}{2}\right) h\right] \times\left[\left(l-\frac{1}{2}\right) h,\left(l+\frac{1}{2}\right) h\right]=\left[x_{k}-\frac{h}{2}, x_{k}+\frac{h}{2}\right] \times\left[y_{l}-\frac{h}{2}, y_{l}+\frac{h}{2}\right],
$$

where, $h>0$ is the mesh size parameter, $k, l \in \mathbb{Z}$. Let us denote by $E(s):\left(L^{2}\left(\mathbb{R}^{2}\right)\right)^{p} \rightarrow$ $\left(L^{2}\left(\mathbb{R}^{2}\right)\right)^{p}$ the exact evolution operator for a general hyperbolic system $(2.1)$, i.e.

$$
\mathbf{U}(., t+s)=E(s) \mathbf{U}(., t) .
$$

We suppose that $S_{h}^{m}$ is a finite element space consisting of piecewise polynomials of degree $m \geq 0$ with respect to the square mesh. Assume a constant time step, i.e. $t_{n}=n \Delta t$. Let $\mathbf{U}^{n}$ be an approximation in the space $S_{h}^{m}$ to the exact solution $\mathbf{U}\left(., t_{n}\right)$ at time $t_{n} \geq 0$. We consider $E_{\tau}:\left(L^{2}\left(\mathbb{R}^{2}\right)\right)^{p} \rightarrow\left(L^{2}\left(\mathbb{R}^{2}\right)\right)^{p}$ to be a suitable approximate evolution operator for $E(\tau)$. In practice we will use restrictions of $E_{\tau}$ to the subspace $S_{h}^{m}$ for $m \geq 0$. Then we can define the general class of evolution Galerkin methods as follows. 
Definition 4.2 Starting from some initial data $\mathbf{U}^{0} \in S_{h}^{m}$ at time $t=0$, an evolution Galerkin method (EG-method) is recursively defined by means of

$$
\mathbf{U}^{n+1}=P_{h} E_{\tau} \mathbf{U}^{n},
$$

where $P_{h}$ is the $L^{2}$-projection given by the integral averages in the following way:

$$
\left.P_{h} \mathbf{U}^{n}\right|_{\Omega_{k l}}=\frac{1}{\left|\Omega_{k l}\right|} \int_{\Omega_{k l}} \mathbf{U}\left(x, y, t_{n}\right) d x d y
$$

In this paper we will limit our considerations to the cases where $m=0$. In this case the integrals that we obtain from the projection are evaluated either exactly using the fact that the approximate values $\mathbf{U}^{n}$ are piecewise constant or by means of some numerical quadrature rules. Using piecewise constants, the resulting schemes will only be of first order accuracy, even when $E_{\tau}$ is approximated to a higher order. Higher order accuracy can be obtained either by taking $m>0$, or by inserting a recovery stage $R_{h}$ before the evolution step in equation (4.3) to give

$$
\mathbf{U}^{n+1}=P_{h} E_{\tau} R_{h} \mathbf{U}^{n} .
$$

Here we have denoted by $R_{h}: S_{h}^{m} \rightarrow S_{h}^{r}$ a recovery operator, $r>m \geq 0$ and consider our approximate evolution operator $E_{\tau}$ on $S_{h}^{r}$. To implement (4.5) rather complex threedimensional integrals need to be evaluated exactly. This approach seems to be hardly feasible for efficient derivation and implementation of higher order methods. A simplification that we used is to apply the multidimensional evolution only on the cell interfaces. This latter approach leads to the finite volume evolution Galerkin methods.

Definition 4.6 Starting from some initial data $\mathbf{U}^{0} \in S_{h}^{m}$, the finite volume evolution Galerkin method (FVEG) is recursively defined by means of

$$
\mathbf{U}^{n+1}=\mathbf{U}^{n}-\frac{1}{h} \int_{0}^{\Delta t} \sum_{j=1}^{2} \delta_{x_{j}} \mathbf{f}_{j}\left(\tilde{\mathbf{U}}^{n+\frac{\tau}{\Delta t}}\right) d \tau,
$$

where $\delta_{x_{j}} \mathbf{f}_{j}\left(\tilde{\mathbf{U}}^{n+\frac{\tau}{\Delta t}}\right)$ represents an approximation to the edge flux difference and $\delta_{x}$ is defined by $\delta_{x} v(x)=v\left(x+\frac{h}{2}\right)-v\left(x-\frac{h}{2}\right)$. The cell boundary value $\tilde{\mathbf{U}}^{n+\frac{\tau}{\Delta t}}$ is evolved using the approximate evolution operator $E_{\tau}$ to $t_{n}+\tau$ and averaged along the cell boundary, i.e.

$$
\tilde{\mathbf{U}}^{n+\frac{\tau}{\Delta t}}=\sum_{k, l \in \mathbb{Z}}\left(\frac{1}{\left|\partial \Omega_{k l}\right|} \int_{\partial \Omega_{k l}} E_{\tau} R_{h} \mathbf{U}^{n} d S\right) \chi_{k l}
$$

where $\chi_{k l}$ is the characteristic function of $\partial \Omega_{k l}$.

For more details on higher order finite volume evolution Galerkin (FVEG) schemes, see [10], [15], [17], where the error analysis as well as numerical experiments are presented. Using the $L^{2}$-projection (4.4), the approximate evolution operator $E_{\tau}$, and (4.7), (4.8) the EG and the FVEG schemes can be written in the finite difference form

$$
\mathbf{U}_{k l}^{n+1}=\mathbf{U}_{k l}^{n}+\sum_{r=1}^{1} \sum_{s=-1}^{1} \mathcal{C}_{r s} \mathbf{U}_{k+r, l+s}^{n},
$$


where

$$
\mathcal{C}_{r s}=\left(\begin{array}{ccc}
\alpha_{r s}^{1} & \beta_{r s}^{1} & \gamma_{r s}^{1} \\
\alpha_{r s}^{2} & \beta_{r s}^{2} & \gamma_{r s}^{2} \\
\alpha_{r s}^{3} & \beta_{r s}^{3} & \gamma_{r s}^{3}
\end{array}\right)
$$

Here the entries $\alpha_{r s}^{m}, \beta_{r s}^{m}, \gamma_{r s}^{m}, m=1,2,3$, are chosen appropriately according to the approximate evolution operator $E_{\tau}$ used. In the Appendix the stencil matrices $\alpha^{m}, \beta^{m}$ and $\gamma^{m}$, $m=1,2,3$, are displayed for some EG schemes.

\section{Basic tools}

As we mentioned above our stability considerations are based on Fourier analysis. We first recall some basic concepts. Let $\left\{\psi_{k l}^{n}\right\}_{k, l=-\infty}^{\infty}$ be a two dimensional sequence in $\ell_{2}$.

Definition 5.1 The discrete Fourier transformation of $\left\{\psi_{k l}^{n}\right\} \in \ell_{2}$ is the function $\hat{\psi}^{n} \in$ $L_{2}\left(\left[-\frac{\pi}{h}, \frac{\pi}{h}\right] \times\left[-\frac{\pi}{h}, \frac{\pi}{h}\right]\right)$ defined by

$$
\hat{\psi}^{n}(\xi, \eta)=h^{2} \sum_{k=-\infty}^{\infty} \sum_{l=-\infty}^{\infty} \psi_{k l}^{n} \exp ^{-i h(k \xi+l \eta)} .
$$

Similarly to the continuous Fourier transform, we have both an inverse formula and Parseval's identity.

Lemma 5.2 (Inverse formula) If $\left\{\psi_{k l}^{n}\right\} \in \ell_{2}$ and $\hat{\psi}^{n}$ is the discrete Fourier transform of $\left\{\psi_{k l}^{n}\right\}$, then

$$
\psi_{k l}^{n}=\frac{1}{4 \pi^{2}} \int_{-\frac{\pi}{h}}^{\frac{\pi}{h}} \int_{-\frac{\pi}{h}}^{\frac{\pi}{h}} \hat{\psi}^{n}(\xi, \eta) \exp ^{i h(k \xi+l \eta)} d \xi d \eta .
$$

Lemma 5.3 (Parseval's identity) If $\left\{\psi_{k l}^{n}\right\} \in \ell_{2}$ and $\hat{\psi}^{n}$ is the discrete Fourier transform of $\left\{\psi_{k l}^{n}\right\}$, then

$$
\left\|\hat{\psi}^{n}\right\|=\left\|\psi_{k l}^{n}\right\|,
$$

where the first norm is the $L_{2}$-norm on $\left[-\frac{\pi}{h}, \frac{\pi}{h}\right] \times\left[-\frac{\pi}{h}, \frac{\pi}{h}\right]$ and the second norm is the $\ell_{2}$-norm.

Hence we have the following result.

Lemma 5.4 The sequence $\left\{\psi_{k l}^{n}\right\}$ is bounded in $\ell_{2}$ if and only if the sequence $\left\{\hat{\psi}^{n}\right\}$ is bounded in $L_{2}\left(\left[-\frac{\pi}{h}, \frac{\pi}{h}\right] \times\left[-\frac{\pi}{h}, \frac{\pi}{h}\right]\right)$.

In order to study the stability of linear numerical schemes the Fourier transform is used. This leads to a bound on the spectral radius of the so-called amplification matrix. The spectral radius of a square complex matrix $\mathcal{A}$ with eigenvalues $\lambda_{i}$ is defined to be

$$
\rho(\mathcal{A})=\max _{i}\left|\lambda_{i}\right| .
$$

The spectral norm of the matrix $\mathcal{A}$ is defined as

$$
\|\mathcal{A}\|=\sup _{\mathbf{x} \neq 0} \frac{\|\mathcal{A} \mathbf{x}\|}{\|\mathbf{x}\|} .
$$

The norms on the right-hand side of equation (5.6) are the Euclidean norms of the vectors $\mathcal{A} \mathrm{x}$ and $\mathrm{x}$, respectively. Note that for the spectral norm, as for any matrix norm, we always have $\|\mathcal{A}\| \geq \rho(\mathcal{A})$. 


\section{Estimate of the stability limit}

In [13, Lemma 5.1] Lukáčová et al. proved the following stability result for the EG-schemes. There exists $\nu_{\max }<1$ such that EG schemes for the two-dimensional wave equation system (3.1) are stable for any $\nu$ such that $0 \leq \nu \leq \nu_{\max }$, where $\nu=c \frac{\Delta t}{h}$ is the CFL number. The goal of this section is to estimate $\nu_{\max }$ for the EG3 scheme by means of a von Neumann stability analysis. We refer to [2] for a related approach used to estimate stability limits of other finite volume schemes for the Maxwell equations. Analogous calculations can be done also for other EG-schemes of type EG1, EG2, EG4 as well as for the FVEG schemes. First we apply the discrete Fourier transform to both sides of equation (4.9).

$$
\hat{\mathbf{U}}^{n+1}=\hat{\mathbf{U}}^{n}+h^{2} \sum_{k=-\infty}^{\infty} \sum_{l=-\infty}^{\infty}\left(\sum_{r=-1}^{1} \sum_{s=-1}^{1} \mathcal{C}_{r s} \mathbf{U}_{k+r l+s}^{n}\right) \exp ^{-i h(k \xi+l \eta)} .
$$

By making the change of variables $k^{\prime}=k+r$ and $l^{\prime}=l+s$ we get

$$
\begin{aligned}
h^{2} \sum_{k=-\infty}^{\infty} \sum_{l=-\infty}^{\infty} & \left(\sum_{r=-1}^{1} \sum_{s=-1}^{1} \mathcal{C}_{r s} \mathbf{U}_{k+r l+s}^{n}\right) \exp ^{-i h(k \xi+l \eta)} \\
= & \sum_{r=-1}^{1} \sum_{s=-1}^{1} \mathcal{C}_{r s} \exp ^{i h(r \xi+s \eta)}\left(h^{2} \sum_{k^{\prime}=-\infty}^{\infty} \sum_{l^{\prime}=-\infty}^{\infty} \mathbf{U}_{k^{\prime} l^{\prime}}^{n} \exp ^{-i h\left(k^{\prime} \xi+l^{\prime} \eta\right)}\right) \\
= & \sum_{r=-1}^{1} \sum_{s=-1}^{1} \mathcal{C}_{r s} \exp ^{i h(r \xi+s \eta)} \hat{\mathbf{U}}^{n} .
\end{aligned}
$$

Thus, using this expression in the equation (6.1), we get

$$
\hat{\mathbf{U}}^{n+1}=\left(\mathcal{I}+\sum_{r=-1}^{1} \sum_{s=-1}^{1} \mathcal{C}_{r s} \exp ^{i h(r \xi+s \eta)}\right) \hat{\mathbf{U}}^{n},
$$

where $\mathcal{I}$ is the identity matrix. The coefficient of $\hat{\mathbf{U}}^{n}$ in the equation (6.3),

$$
\mathcal{T}(\xi, \eta)=\mathcal{I}+\sum_{r=-1}^{1} \sum_{s=-1}^{1} \mathcal{C}_{r s} \exp ^{i h(r \xi+s \eta)},
$$

is called the amplification matrix of the finite difference scheme (4.9). Applying recursively the result of equation (6.3) $n+1$ times yields

$$
\hat{\mathbf{U}}^{n+1}=\left(\mathcal{I}+\sum_{r=-1}^{1} \sum_{s=-1}^{1} \mathcal{C}_{r s} \exp ^{i h(r \xi+s \eta)}\right)^{n+1} \hat{\mathbf{U}}^{0}=\mathcal{T}^{n+1}(\xi, \eta) \hat{\mathbf{U}}^{0} .
$$

We note that if $\|\mathcal{T}(\xi, \eta)\| \leq 1$ then $\left\|\hat{\mathbf{U}}^{n+1}\right\| \leq\left\|\hat{\mathbf{U}}^{0}\right\|$, which means that the $\left\{\hat{\mathbf{U}}^{n}\right\}$ is $L^{2}$-stable. Consider the EG3 scheme, i.e. the numerical scheme based on equations (3.7) - (3.9), cf. also the stencil matrices in the Appendix. After some calculation we obtain the entries of the amplification matrix $\mathcal{T}(\xi, \eta)$ :

$$
\begin{aligned}
& T_{11}(\xi, \eta)=1+\frac{\nu^{2}}{\pi}-\frac{4 \nu}{\pi}+\frac{\nu^{2}}{\pi} \cos (h \xi) \cos (h \eta)+\left(\frac{2 \nu}{\pi}-\frac{\nu^{2}}{\pi}\right)(\cos (h \xi)+\cos (h \eta)), \\
& T_{12}(\xi, \eta)=-i\left(\frac{4 \nu^{2}}{3 \pi} \sin (h \xi) \cos (h \eta)+\left(\nu-\frac{4 \nu^{2}}{3 \pi}\right) \sin (h \xi)\right),
\end{aligned}
$$




$$
\begin{aligned}
& T_{13}(\xi, \eta)=-i\left(\frac{4 \nu^{2}}{3 \pi} \cos (h \xi) \sin (h \eta)+\left(\nu-\frac{4 \nu^{2}}{3 \pi}\right) \sin (h \eta)\right) \\
& T_{22}(\xi, \eta)=1-\frac{2 \nu}{\pi}+\frac{\nu^{2}}{2 \pi}+\frac{\nu^{2}}{2 \pi} \cos (h \xi) \cos (h \eta)+\left(\frac{2 \nu}{\pi}-\frac{\nu^{2}}{2 \pi}\right) \cos (h \xi)-\frac{\nu^{2}}{2 \pi} \cos (h \eta), \\
& T_{23}(\xi, \eta)=\frac{-3 \nu^{2}}{8} \sin (h \xi) \sin (h \eta), \\
& T_{33}(\xi, \eta)=1-\frac{2 \nu}{\pi}+\frac{\nu^{2}}{2 \pi}+\frac{\nu^{2}}{2 \pi} \cos (h \xi) \cos (h \eta)+\left(\frac{2 \nu}{\pi}-\frac{\nu^{2}}{2 \pi}\right) \cos (h \eta)-\frac{\nu^{2}}{2 \pi} \cos (h \xi), \\
& T_{21}(\xi, \eta)=T_{12}(\xi, \eta), \quad T_{31}(\xi, \eta)=T_{13}(\xi, \eta), \quad T_{32}(\xi, \eta)=T_{23}(\xi, \eta) .
\end{aligned}
$$

Using the substitutions $S_{\xi}=\sin (h \xi), s_{\xi}=\sin \left(\frac{h \xi}{2}\right), S_{\eta}=\sin (h \eta)$ and $s_{\eta}=\sin \left(\frac{h \eta}{2}\right)$ the amplification matrix $\mathcal{T}=\mathcal{T}(\xi, \eta)$ can be written as

$$
\mathcal{T}=\left(\begin{array}{lll}
C_{11} & -i \nu C_{\xi} & -i \nu C_{\eta} \\
-i \nu C_{\xi} & C_{22} & \nu^{2} C_{\xi \eta} \\
-i \nu C_{\eta} & \nu^{2} C_{\xi \eta} & C_{33}
\end{array}\right)
$$

where

$$
\begin{aligned}
C_{11} & =1-\frac{4 \nu}{\pi}\left(s_{\xi}^{2}+s_{\eta}^{2}\right)+\frac{4 \nu^{2}}{\pi} s_{\xi}^{2} s_{\eta}^{2}, \\
C_{\xi} & =S_{\xi}\left(1-\frac{8 \nu}{3 \pi} s_{\eta}^{2}\right), \\
C_{\eta} & =S_{\eta}\left(1-\frac{8 \nu}{3 \pi} s_{\xi}^{2}\right), \\
C_{\xi \eta} & =\frac{-3}{8} S_{\xi} S_{\eta}, \\
C_{22} & =1-\frac{4 \nu}{\pi} s_{\xi}^{2}+\frac{2 \nu^{2}}{\pi} s_{\xi}^{2} s_{\eta}^{2}, \\
C_{33} & =1-\frac{4 \nu}{\pi} s_{\eta}^{2}+\frac{2 \nu^{2}}{\pi} s_{\xi}^{2} s_{\eta}^{2} .
\end{aligned}
$$

Set $\mathcal{E}=\left(\begin{array}{ccc}i & 0 & 0 \\ 0 & 1 & 0 \\ 0 & 0 & 1\end{array}\right)$, then $\mathcal{Q}=\left(\begin{array}{lll}C_{11} & -\nu C_{\xi} & -\nu C_{\eta} \\ \nu C_{\xi} & C_{22} & \nu^{2} C_{\xi \eta} \\ \nu C_{\eta} & \nu^{2} C_{\xi \eta} & C_{33}\end{array}\right)=\mathcal{E}^{-1} \mathcal{T} \mathcal{E}$, which means that $\mathcal{T}$ and $\mathcal{Q}$ are similar matrices and thus they have the same eigenvalues. Moreover, the matrix $\mathcal{Q}$ can be decomposed as

$$
\mathcal{Q}=\mathcal{I}-\nu(\mathcal{D}+\mathcal{C})+\nu^{2} \tilde{\mathcal{C}}
$$

where

$$
\begin{aligned}
\mathcal{D} & =\left(\begin{array}{lll}
d+f & 0 & 0 \\
0 & d & 0 \\
0 & 0 & f
\end{array}\right), \quad \mathcal{C}=\left(\begin{array}{lll}
0 & C_{\xi} & C_{\eta} \\
-C_{\xi} & 0 & 0 \\
-C_{\eta} & 0 & 0
\end{array}\right), \quad \tilde{\mathcal{C}}=\left(\begin{array}{lll}
0 & 0 & 0 \\
0 & 0 & C_{\xi \eta} \\
0 & C_{\xi \eta} & 0
\end{array}\right), \\
d & =\frac{4}{\pi} s_{\xi}^{2}-\frac{2 \nu}{\pi} s_{\xi}^{2} s_{\eta}^{2}=\frac{2}{\pi} s_{\xi}^{2}\left(2-\nu s_{\eta}^{2}\right), \quad f=\frac{4}{\pi} s_{\eta}^{2}-\frac{2 \nu}{\pi} s_{\xi}^{2} s_{\eta}^{2}=\frac{2}{\pi} s_{\eta}^{2}\left(2-\nu s_{\xi}^{2}\right) .
\end{aligned}
$$

Let

$$
\mathcal{H}=\mathcal{I}-\nu(\mathcal{D}+\mathcal{C})
$$


and $\|\cdot\|_{\star}$ be an operator norm such that $\|\mathcal{H}\|_{\star}=\|\mathcal{J}\|_{\infty}$, where $\mathcal{J}$ is a scaled Jordan normal form of $\mathcal{H}$ having $\epsilon<<1$ on the first off-diagonal and eigenvalues of $\mathcal{H}$ on the diagonal. According to [5] we know that the following property holds: $\rho(\mathcal{H})<1$ if and only if $\|\mathcal{H}\|_{\star}<1$. Since all norms in finitely-dimensional spaces are equivalent we have

$$
\|\mathcal{Q}-(\mathcal{I}-\nu(\mathcal{D}+\mathcal{C}))\|_{\star} \leq c|| \mathcal{Q}-(\mathcal{I}-\nu(\mathcal{D}+\mathcal{C})) \|=c \nu^{2}\left|C_{\xi \eta}\right|=O\left(\nu^{2}\right) .
$$

Thus, using (6.7) we obtain

$$
\rho(\mathcal{Q}) \leq\|Q\|_{\star} \leq\|\mathcal{H}\|_{\star}+c \nu^{2},
$$

and it suffices to study the spectrum of matrix $\mathcal{H}$, since $\rho(\mathcal{H})<1$ if and only if $\|\mathcal{H}\|_{\star}<1$. Further, since $\mathcal{H}=\mathcal{I}-\nu(\mathcal{D}+\mathcal{C})$ and due to the form of $\mathcal{D}, \mathcal{C}$ it can be shown readily that $\|\mathcal{H}\|_{\infty}=\left|1+c_{1} \nu+c_{2} \nu^{2}\right|$ for some constants $c_{1}, c_{2} \in \mathbb{R}$. Since all norms in finitely-dimensional spaces are equivalent, there exist $k_{1}, k_{2}>0$, such that for any matrix $\mathcal{M} \in \mathbb{R}^{(3,3)}$

$$
k_{1}\|\mathcal{M}\|_{\infty} \leq\|\mathcal{M}\|_{\star} \leq k_{2}\|\mathcal{M}\|_{\infty}
$$

In particular, if $\mathcal{M}=\mathcal{H}$ we have for any $\nu>0$

$$
k_{1}\left|1+c_{1} \nu+c_{2} \nu^{2}\right| \leq\|\mathcal{H}\|_{\star} \leq k_{2}\left|1+c_{1} \nu+c_{2} \nu^{2}\right| .
$$

Thus, it is clear that $\|\mathcal{H}\|_{\star}$ depends at most quadratically on $\nu$, i.e. $\|\mathcal{H}\|_{\star}=\left|1+c_{3} \nu+c_{4} \nu^{2}\right|$. Now, if we assume that $\|\mathcal{H}\|_{\star}<1$, the linear term has to be negative, i.e. $c_{3}<0$, as otherwise $\|\mathcal{H}\|_{\star}$ cannot be strictly less than 1 for $\nu \leq 1$. Thus, if $\|\mathcal{H}\|_{\star}<1$ we can find small enough $\nu$ such that $\|\mathcal{H}\|_{\star}+c \nu^{2} \leq 1$ and $\rho(Q) \leq 1$ due to (6.8).

Unfortunately, we cannot give any quantitative estimate on $\nu$ since we do not know how large the constant $c$ in (6.8) is. However, we know from [13] that there exists $\nu_{\max }>0$ such that for all $\nu \in\left(0, \nu_{\max }\right]$ we have $\rho(Q) \leq 1$.

In what follows we will study the spectrum of the matrix $\mathcal{H}$ and find $\nu$ such that $\rho(\mathcal{H})<1$. Note that for all $(\xi, \eta) \in\left[\frac{-\pi}{h}, \frac{\pi}{h}\right] \times\left[\frac{-\pi}{h}, \frac{\pi}{h}\right]$ the entries of $\mathcal{H}$ are bounded. We need to estimate the spectral radius of $\mathcal{H}$ for all choices of $\xi, \eta$ and $\nu, 0<\nu \leq 1$.

First of all, it is easy to see that in a special case when $\xi=0=\eta$ we have $d=f=C_{\xi}=$ $C_{\eta}=C_{\xi \eta}=0$ and $\mathcal{Q}=\mathcal{I}=\mathcal{H}$. Thus, trivially $\rho(\mathcal{H})=\rho(\mathcal{Q})=1$ for any $\nu$. Therefore in what follows it suffices to study the case, when $\xi \neq 0$ or $\eta \neq 0$.

Since $0 \leq s_{\xi}^{2} \leq 1$ and $0 \leq s_{\eta}^{2} \leq 1$ and $\nu \leq 1$ then $d \geq 0$ and $f \geq 0$. Now the matrices $\mathcal{D}, \mathcal{C}$ are real and $\mathcal{C}$ is skew symmetric. Hence $\mathcal{D}+\mathcal{C}$ has either three real eigenvalues or one real eigenvalue and two complex conjugate eigenvalues.

Consider a real eigenvalue, say $\lambda=\lambda_{r}$. Let $\mathbf{v}=\left(v_{1}, v_{2}, v_{3}\right)$ be the corresponding eigenvector; then $\mathbf{v}^{T}(\mathcal{D}+\mathcal{C}) \mathbf{v}=\mathbf{v}^{T} \lambda_{r} \mathbf{v}$. Since $\mathcal{C}$ is skew symmetric then $\mathbf{v}^{T} \mathcal{C} \mathbf{v}=0$. Hence we get

$$
\left(d+f-\lambda_{r}\right) v_{1}^{2}+\left(d-\lambda_{r}\right) v_{2}^{2}+\left(f-\lambda_{r}\right) v_{3}^{2}=0 .
$$

The coefficients in equation (6.10) cannot all have the same sign for $v_{1}^{2}, v_{2}^{2}, v_{3}^{2}>0$. Therefore, we get the estimates

$$
0 \leq \min (d, f) \leq \lambda_{r} \leq d+f .
$$


Let $\mu_{r}$ be a real eigenvalue of $\mathcal{H}$; then $\mu_{r}=1-\nu \lambda_{r}$. Hence $\left|\mu_{r}\right|<1$ is equivalent to $-1<1-\nu \lambda_{r}<1$. According to the inequality (6.11) we assume now that

$$
\lambda_{r}>0
$$

the case $\lambda_{r}=0$ will be treated separately later, cf. (6.22). Further,

$$
1-\frac{4 \nu}{\pi}\left(s_{\xi}^{2}+s_{\eta}^{2}\right)+\frac{4 \nu^{2}}{\pi} s_{\xi}^{2} s_{\eta}^{2} \leq 1-\nu \lambda_{r}<1 .
$$

To ensure that $\left|\mu_{r}\right|<1$ we need

$$
1-\frac{4 \nu}{\pi}\left(s_{\xi}^{2}+s_{\eta}^{2}\right)+\frac{4 \nu^{2}}{\pi} s_{\xi}^{2} s_{\eta}^{2}>-1
$$

The last inequality reads

$$
\nu^{2}\left(\frac{4}{\pi} s_{\xi}^{2} s_{\eta}^{2}\right)-\nu\left(\frac{4}{\pi}\left(s_{\xi}^{2}+s_{\eta}^{2}\right)\right)+2>0
$$

It suffices to bound $\nu$ so that $2-\nu \frac{4}{\pi}\left(s_{\xi}^{2}+s_{\eta}^{2}\right)>0$. Since $\left(s_{\xi}^{2}+s_{\eta}^{2}\right) \leq 2$, this is true if

$$
\nu<\frac{\pi}{4} \approx 0.7854
$$

Now let us assume that $\mu_{c}$ is a complex eigenvalue of $\mathcal{H}$. Then $\mu_{c}=1-\nu \lambda_{c}$, where $\lambda_{c}$ is a complex eigenvalue of the matrix $\mathcal{D}+\mathcal{C}$. This implies that

$$
\left|\mu_{c}\right|^{2}=1-2 \nu \operatorname{Re}\left(\lambda_{c}\right)+\nu^{2}\left|\lambda_{c}\right|^{2} .
$$

Thus $\left|\mu_{c}\right|^{2}<1$ is equivalent to $\nu^{2}\left|\lambda_{c}\right|^{2}-2 \nu \operatorname{Re}\left(\lambda_{c}\right)<0$. Since $\lambda_{r}>0$, cf. (6.12), we have

$$
\nu^{2} \lambda_{r}\left|\lambda_{c}\right|^{2}-2 \nu \lambda_{r} \operatorname{Re}\left(\lambda_{c}\right)<0 .
$$

Let $b=C_{\xi}$ and $c=C_{\eta}$. It is well known that

$$
\begin{aligned}
\operatorname{det}(\mathcal{D}+\mathcal{C}) & =d^{2} f+f^{2} d+b^{2} f+c^{2} d=\lambda_{r}\left|\lambda_{c}\right|^{2} \\
\operatorname{Tr}(\mathcal{D}+\mathcal{C}) & =2(d+f)=\lambda_{r}+\lambda_{c}+\bar{\lambda}_{c}=\lambda_{r}+2 \operatorname{Re}\left(\lambda_{c}\right),
\end{aligned}
$$

Hence inequality (6.15) reads

$$
p\left(\lambda_{r}\right)=\lambda_{r}^{2}-2(d+f) \lambda_{r}+\nu\left(d^{2} f+f^{2} d+b^{2} f+c^{2} d\right)<0 .
$$

Let us consider the polynomial

$$
p=p(\lambda)=\lambda^{2}-2(d+f) \lambda+\nu\left(d^{2} f+f^{2} d+b^{2} f+c^{2} d\right) .
$$

The discriminant of $p$ gives

$$
\Delta=4(d+f)^{2}-4 \nu\left(d^{2} f+f^{2} d+b^{2} f+c^{2} d\right)=4\left(d^{2}+f^{2}\right)+8 f d-4 \nu\left(d^{2} f+f^{2} d+b^{2} f+c^{2} d\right) .
$$


It suffices to show that the following inequality holds

$$
8 f d-4 \nu\left(d^{2} f+f^{2} d+b^{2} f+c^{2} d\right)>0
$$

which leads to $\Delta>4\left(d^{2}+f^{2}\right) \geq 0$. Now

$$
\begin{aligned}
8 f d=\frac{32}{\pi^{2}} s_{\xi}^{2} s_{\eta}^{2}\left(2-\nu s_{\eta}^{2}\right)\left(2-\nu s_{\xi}^{2}\right) & =\frac{32}{\pi^{2}} s_{\xi}^{2} s_{\eta}^{2}\left(4-2 \nu\left(s_{\xi}^{2}+s_{\eta}^{2}\right)+\nu^{2} s_{\xi}^{2} s_{\eta}^{2}\right) \\
& \geq \frac{32}{\pi^{2}} s_{\xi}^{2} s_{\eta}^{2}\left(4-2 \nu\left(s_{\xi}^{2}+s_{\eta}^{2}\right)\right) .
\end{aligned}
$$

Note that the last inequality is strict if $\xi \neq 0$ and $\eta \neq 0$. Hence,

$$
8 f d \geq \frac{32}{\pi^{2}} s_{\xi}^{2} s_{\eta}^{2}(4-4 \nu)=\frac{128}{\pi^{2}} s_{\xi}^{2} s_{\eta}^{2}(1-\nu) .
$$

If $\xi \neq 0$ and $\eta \neq 0$ the inequality in (6.17) is strict.

Further, we have

$$
\begin{aligned}
& d^{2} f=\frac{8}{\pi^{3}} s_{\xi}^{4} s_{\eta}^{2}\left(2-\nu s_{\eta}^{2}\right)^{2}\left(2-\nu s_{\xi}^{2}\right) \leq \frac{64}{\pi^{3}} s_{\xi}^{4} s_{\eta}^{2} \leq \frac{64}{\pi^{3}} \\
& b^{2} f=S_{\xi}^{2}\left(1-\frac{8 \nu^{2}}{3 \pi} s_{\eta}^{2}\right)^{2} \frac{2}{\pi} s_{\eta}^{2}\left(2-\nu s_{\xi}^{2}\right) \leq \frac{4}{\pi} S_{\xi}^{2} s_{\eta}^{2} \leq \frac{4}{\pi}
\end{aligned}
$$

Again, note that in the case that either $\xi=0$ or $\eta=0$ the inequality in (6.18) is strict.

Analogously, we obtain

$$
f^{2} d \leq \frac{64}{\pi^{3}} \quad \text { and } \quad c^{2} d \leq \frac{4}{\pi}
$$

Therefore,

$$
-4 \nu\left(d^{2} f+f^{2} d+b^{2} f+c^{2} d\right) \geq-4 \frac{128+8 \pi^{2}}{\pi^{3}} \nu ;
$$

if $\xi=0$ or $\eta=0$ the above inequality is strict.

Combining inequalities (6.17) and (6.20) we get

$$
\begin{aligned}
8 d f-4 \nu\left(d^{2} f+f^{2} d+b^{2} f+c^{2} d\right) & >\frac{128}{\pi^{2}} s_{\xi}^{2} s_{\eta}^{2}(1-\nu)-4\left(\frac{128+8 \pi^{2}}{\pi^{3}}\right) \nu \\
& =\frac{128}{\pi^{2}} s_{\xi}^{2} s_{\eta}^{2}-\nu\left(\frac{128}{\pi^{2}} s_{\xi}^{2} s_{\eta}^{2}+4\left(\frac{128+8 \pi^{2}}{\pi^{3}}\right)\right) \geq 0 .
\end{aligned}
$$

The last inequality implies

$$
\nu \leq \frac{\frac{128}{\pi^{2}} s_{\xi}^{2} s_{\eta}^{2}}{4\left(\frac{128+8 \pi^{2}}{\pi^{3}}\right)+\frac{128}{\pi^{2}} s_{\xi}^{2} s_{\eta}^{2}} \leq \frac{\frac{128}{\pi^{2}}}{4\left(\frac{128+8 \pi^{2}}{\pi^{3}}\right)+\frac{128}{\pi^{2}} s_{\xi}^{2} s_{\eta}^{2}}
$$

Since

$$
4\left(\frac{128+8 \pi^{2}}{\pi^{3}}\right)+\frac{128}{\pi^{2}} s_{\xi}^{2} s_{\eta}^{2} \geq 4\left(\frac{128+8 \pi^{2}}{\pi^{3}}\right)
$$

we then have

$$
\frac{1}{4\left(\frac{128+8 \pi^{2}}{\pi^{3}}\right)+\frac{128}{\pi^{2}} s_{\xi}^{2} s_{\eta}^{2}} \leq \frac{1}{4\left(\frac{128+8 \pi^{2}}{\pi^{3}}\right)} .
$$


Therefore we get

$$
\nu \leq \frac{\frac{128}{\pi^{2}}}{\frac{4\left(128+8 \pi^{2}\right)}{\pi^{3}}}=\frac{32 \pi}{128+8 \pi^{2}} \approx 0.4858 .
$$

Thus we have obtained a sufficient condition on $\nu$ for $\Delta>0$. For $\nu \leq 0.4858$ we have $\Delta>4\left(d^{2}+f^{2}\right) \geq 0$.

Since $\lambda_{r}>0$, cf. (6.12), $p(\lambda)$ has two distinct real roots $r_{1}$ and $r_{2}$, where

$$
r_{1}=(d+f)-\frac{\sqrt{\Delta}}{2}, \quad r_{2}=(d+f)+\frac{\sqrt{\Delta}}{2} .
$$

Inequality (6.11) gives $\lambda_{r}<r_{2}$. To show that $\lambda_{r}>r_{1}$ note that from $\Delta>4\left(d^{2}+f^{2}\right)$ we have $r_{1}<(d+f)-\sqrt{d^{2}+f^{2}}$. Furthermore $\sqrt{d^{2}+f^{2}} \geq \max (d, f)$. Therefore

$$
r_{1}<(d+f)-\sqrt{d^{2}+f^{2}} \leq(d+f)-\max (d, f)=\min (d, f) \leq \lambda_{r} .
$$

Hence $\lambda_{r} \in\left(r_{1}, r_{2}\right)$. This implies that $p\left(\lambda_{r}\right)<0$, as we wished to show, cf. (6.16).

Since $\lambda_{r} \geq 0$, cf. (6.11), we need to consider moreover the case $\lambda_{r}=0$. Then either $d=0$ or $f=0$. Suppose $d=\frac{2}{\pi} s_{\xi}^{2}\left(2-\nu s_{\eta}^{2}\right)=0$; the case $f=0$ is analogous. Then we have that $s_{\xi}=0, \xi=0$ and

$$
\mathcal{D}+\mathcal{C}=\left(\begin{array}{lll}
\frac{4}{\pi} s_{\eta}^{2} & 0 & S_{\eta} \\
0 & 0 & 0 \\
-S_{\eta} & 0 & \frac{4}{\pi} s_{\eta}^{2}
\end{array}\right) .
$$

Note that in this case $\mathcal{Q}=\mathcal{H}, \mu_{r}=1$ and we need to find the condition on $\nu$ to ensure that $\rho(\mathcal{H})=\rho(\mathcal{Q}) \leq 1$. The eigenvalues of $\mathcal{D}+\mathcal{C}$ are $0, \frac{4}{\pi} s_{\eta}^{2} \pm i S_{\eta}$. Now $\left|\mu_{c}\right|^{2}=\left|1-\nu \lambda_{c}\right|^{2} \leq 1$ gives

$$
\begin{aligned}
\left(1-\nu\left(\frac{4}{\pi} s_{\eta}^{2}+i S_{\eta}\right)\right)\left(1-\nu\left(\frac{4}{\pi} s_{\eta}^{2}-i S_{\eta}\right)\right) & =\left(\left(1-\frac{4}{\pi} \nu s_{\eta}^{2}\right)^{2}+\nu^{2} S_{\eta}^{2}\right) \\
& =1-\frac{8 \nu}{\pi} s_{\eta}^{2}+\frac{16 \nu^{2}}{\pi^{2}} s_{\eta}^{4}+\nu^{2} S_{\eta}^{2} \leq 1 .
\end{aligned}
$$

This leads to

$$
-\frac{8 \nu}{\pi} s_{\eta}^{2}+\nu^{2}\left(\frac{16}{\pi^{2}} s_{\eta}^{4}+S_{\eta}^{2}\right) \leq 0 .
$$

Suppose $s_{\eta} \neq 0$, as otherwise $\eta=0$ and $\xi=0$, which is a special case that has already been considered above. Then we have

$$
\begin{aligned}
-\frac{8}{\pi}+\nu\left(\frac{16}{\pi^{2}} s_{\eta}^{2}+\left(\frac{S_{\eta}}{s_{\eta}}\right)^{2}\right) & \leq 0 \\
\nu\left(\frac{16}{\pi^{2}} s_{\eta}^{2}+\left(\frac{S_{\eta}}{s_{\eta}}\right)^{2}\right) & \leq \frac{8}{\pi} .
\end{aligned}
$$

The last inequality yields

$$
\nu \leq \frac{\frac{8}{\pi}}{\left(\frac{16}{\pi^{2}} s_{\eta}^{2}+\left(\frac{S_{\eta}}{s_{\eta}}\right)^{2}\right)} .
$$


Since

$$
\frac{16}{\pi^{2}} s_{\eta}^{2}+\left(\frac{S_{\eta}}{s_{\eta}}\right)^{2} \leq 4
$$

it suffices to take $\nu$ such that

$$
\nu \leq \frac{2}{\pi} \approx 0.6366
$$

Finally inequalities (6.14), (6.21) and (6.24) imply that if

$$
\nu \leq \frac{32 \pi}{128+8 \pi^{2}} \approx 0.4858
$$

then either $\rho(\mathcal{Q})=\rho(\mathcal{H})=1$ or $\rho(\mathcal{H})<1$. Hence we have proved the following result.

Lemma 6.1 Consider the evolution Galerkin scheme EG3. Then, there exists $\nu$ small enough such that $\rho(\mathcal{T}) \leq 1$, where $\mathcal{T}$ is the amplification matrix of the discrete operator representing the EG3 scheme.

More precisely, we know that $\rho(\mathcal{T}) \leq\|\mathcal{H}\|_{*}+O\left(\nu^{2}\right)$, where $\mathcal{H}$ is the matrix defined in (6.6). Moreover, if $\nu \leq \frac{32 \pi}{128+8 \pi} \approx 0.4858$ then $\rho(\mathcal{H})<1$ and $\|\mathcal{H}\|_{*}<1$, except a special case when $\rho(\mathcal{H})=\rho(\mathcal{T})=1$. Otherwise, there is $\nu$ small enough such that $\rho(\mathcal{T}) \leq 1$.

In the Table 1 we have estimated the stability limit of the scheme EG3 using the standard MATLAB procedure eig for the eigenvalues of the matrix $\mathcal{T}$. Note that our theoretical result $\nu \approx 0.4858$ for a simplified problem gives stronger estimate on the CFL number than the experimental results for the original problem. They show that the EG3 scheme stays stable up to $\nu=0.58$. In Figure 2 left we plot the eigenvalues of the matrix $\mathcal{H}$ as well as the unit

\begin{tabular}{|c|c|}
\hline$\frac{c \Delta t}{h}$ & $\rho_{\xi, \eta}(\mathcal{T}(\xi, \eta))$ for EG3 \\
\hline \hline 0.10 & 1.000000000000000 \\
\hline 0.20 & 1.000000000000000 \\
\hline 0.30 & 1.000000000000000 \\
\hline 0.40 & 1.000000000000000 \\
\hline 0.50 & 1.000000000000000 \\
\hline 0.58 & 1.000000000000000 \\
\hline 0.59 & 1.000003244461521 \\
\hline 0.60 & 1.000112236111448 \\
\hline 0.70 & 1.008474049696319 \\
\hline
\end{tabular}

Table 1: Stability limit using $\rho_{\xi, \eta}(\mathcal{T}(\xi, \eta))$.

circle. A similar plot with different scale is shown in Figure 2 right. In Figure 3 we show, using different scales, the eigenvalues of the amplification matrix corresponding to the first order EG3 scheme. We illustrate that it is possible to include all eigenvalues inside the unit circle for the CFL number up to 0.58. Throughout the paper in order to plot the eigenvalues of amplification matrices we have used $100 \times 100$ values of $h \xi, h \eta \in[-\pi, \pi]$. 

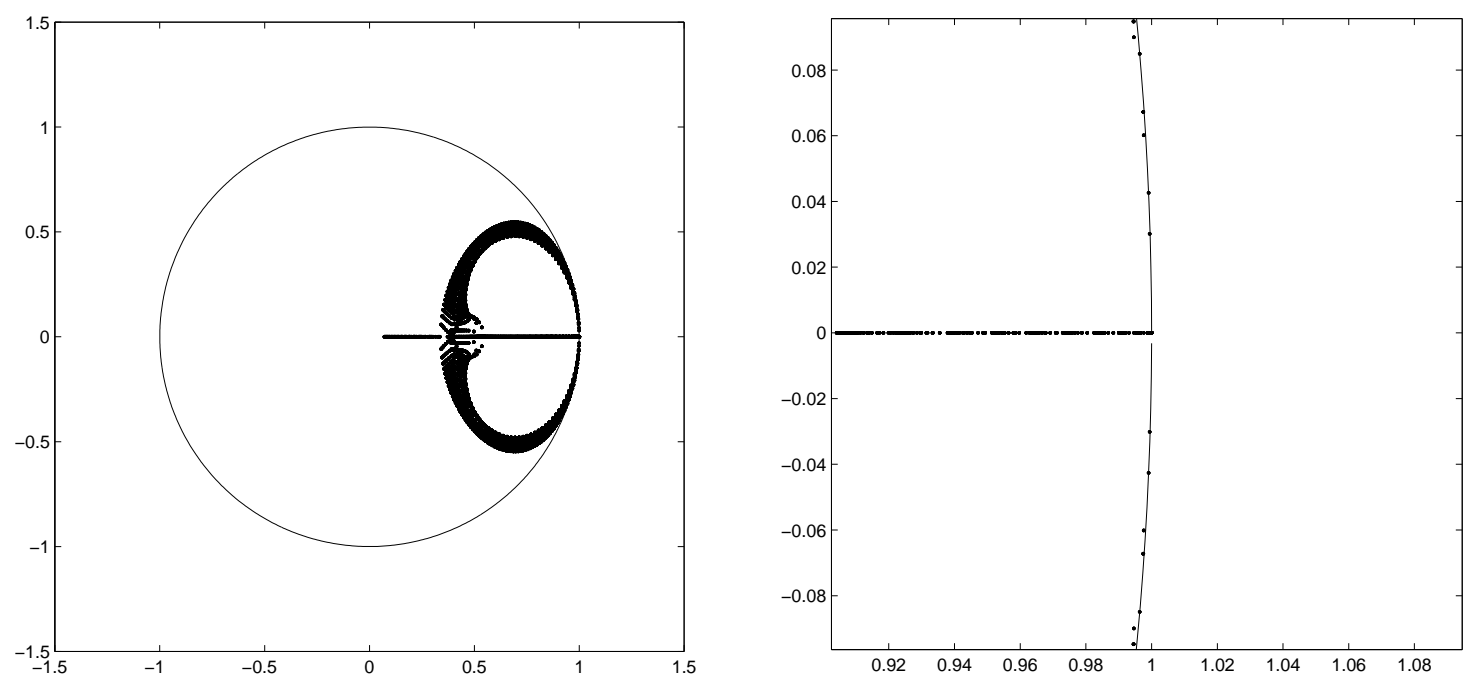

Figure 2: Eigenvalues of the matrix $\mathcal{H}, \mathrm{CFL}=0.48$.
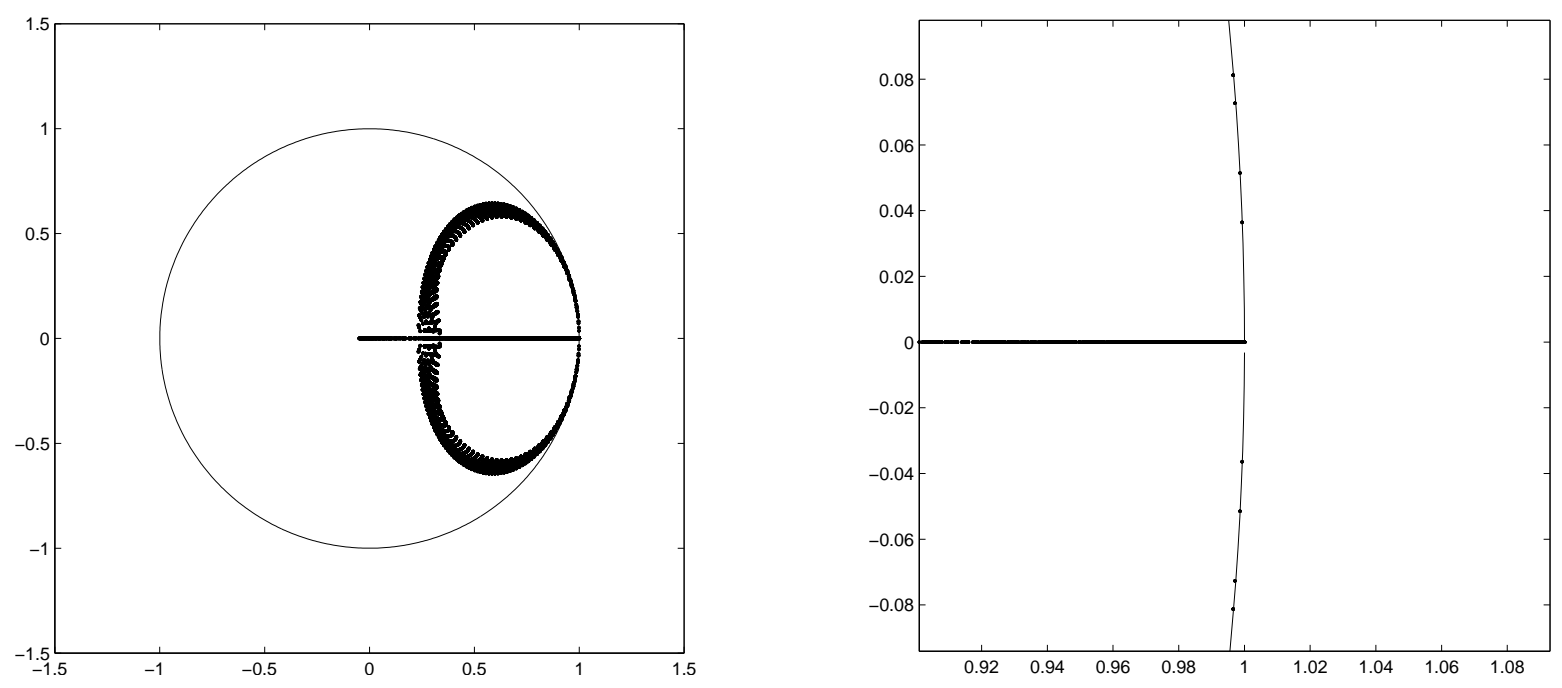

Figure 3: Eigenvalues of the amplification matrix of the first order EG3 scheme for CFL $=0.58$.

\section{Approximate evolution operator $E_{\Delta}^{\text {const }}$ for piecewise con- stant data}

In [11], Lukáčová, Morton and Warnecke proposed new approximate evolution operators $E_{\Delta}^{\text {const }}$ und $E_{\Delta}^{\text {bilin }}$ for the two-dimensional wave equation system and for the Euler equations of gas dynamics. Extensive numerical experiments presented in [11] indicate that these new operators improve the stability of the FVEG-schemes considerably, i.e. in particular, our numerical tests indicated that they have larger stability range than the EG3 method which we considered in Section 6. We will show that for special choices of discretization techniques stability limits close to the natural limit of 1 can be achieved. Numerical experiments, presented in [11], for these FVEG schemes confirm high accuracy as well as good multidimensional behaviour of the new FVEG schemes. The key idea of the development of these new operators 
was to exploit the fact that the exact explicit solution to the one-dimensional wave equation system is available. Our new approximate operators are constructed in such a way that this exact solution is reproduced exactly for a given one-dimensional data. Thus, the approximate evolution operator $E_{\Delta}^{\text {const }}$ calculates exactly any one-dimensional wave which is represented by a piecewise constant data and propagates either in the $x$ - or the $y$ - direction. An analogous situation holds for the operator $E_{\Delta}^{b i l i n}$ and approximated waves by means of continuous piecewise bilinear data. The approximate evolution operator $E_{\Delta}^{\text {const }}$ for piecewise constant data reads, cf. [11]

$$
\begin{aligned}
& \phi_{P}=\frac{1}{2 \pi} \int_{0}^{2 \pi}\left(\phi_{Q}-u_{Q} \operatorname{sgn}(\cos \theta)-v_{Q} \operatorname{sgn}(\sin \theta)\right) \mathrm{d} \theta \\
& u_{P}=\frac{1}{2 \pi} \int_{0}^{2 \pi}\left(-\phi_{Q} \operatorname{sgn}(\cos \theta)+u_{Q}\left(\frac{1}{2}+\cos ^{2} \theta\right)+v_{Q} \sin \theta \cos \theta\right) \mathrm{d} \theta \\
& v_{P}=\frac{1}{2 \pi} \int_{0}^{2 \pi}\left(-\phi_{Q} \operatorname{sgn}(\sin \theta)+u_{Q} \sin \theta \cos \theta+v_{Q}\left(\frac{1}{2}+\sin ^{2} \theta\right)\right) \mathrm{d} \theta .
\end{aligned}
$$

Integrations from 0 to $2 \pi$ around the sonic circle in (7.1) - (7.3) are evaluated exactly. In this way all of the infinitely many directions of wave propagation are taken into account explicitly. For the cell interface integration along $\partial \Omega$ in (4.8) we have two possibilities. These edge integrals can either be computed exactly or numerically. Exact cell interface integration yields, e.g. for the vertical edge, the following intermediate values

$$
\begin{aligned}
\tilde{\Phi}_{\text {edge }}^{n+\frac{1}{2}} & =\left(1+\frac{\nu}{2 \pi} \delta_{y}^{2}\right) \mu_{x} \Phi^{n}-\left(\frac{1}{2}+\frac{\nu}{4 \pi} \delta_{y}^{2}\right) \delta_{x} U^{n}-\frac{\nu}{\pi} \mu_{x} \mu_{y} \delta_{y} V^{n} \\
\tilde{U}_{\text {edge }}^{n+\frac{1}{2}} & =-\left(\frac{1}{2}+\frac{\nu}{4 \pi} \delta_{y}^{2}\right) \delta_{x} \Phi^{n}+\left(1+\frac{5 \nu}{12 \pi} \delta_{y}^{2}\right) \mu_{x} U^{n}+\frac{\nu}{6 \pi} \delta_{x} \mu_{y} \delta_{y} V^{n}
\end{aligned}
$$

where $\mu_{x} f(x)=\frac{1}{2}\left(f\left(x+\frac{h}{2}\right)+f\left(x-\frac{h}{2}\right)\right), \delta_{x}^{2} f(x)=f(x+h)-2 f(x)+f(x-h)$.

The stencil matrices of this FVEG scheme are given in the Appendix. Another possibility to evaluate the cell interface integrals is to use some numerical quadrature. In this way, further simplification in the derivation of the scheme can be made. Instead of the two-dimensional integrals along the cell interfaces and around the sonic circle, only the sonic circle integrals need to be evaluated exactly. In our experiments we used the trapezoidal rule and Simpson's rule for the cell interface integration. Thus, we need to determine $\tilde{\mathbf{U}}^{n+\frac{1}{2}}$ :

$$
\begin{aligned}
\tilde{\Phi}_{\text {vertex }}^{n+\frac{1}{2}} & =\mu_{x} \mu_{y} \Phi^{n}-\frac{1}{2} \mu_{y} \delta_{x} U^{n}-\frac{1}{2} \mu_{x} \delta_{y} V^{n}, \\
\tilde{U}_{\text {vertex }}^{n+\frac{1}{2}} & =-\frac{1}{2} \mu_{y} \delta_{x} \Phi^{n}+\mu_{x} \mu_{y} U^{n}+\frac{1}{4 \pi} \delta_{x} \delta_{y} V^{n}, \\
\tilde{\Phi}_{\text {midpoint }}^{n+\frac{1}{2}} & =\mu_{x} \Phi^{n}-\frac{1}{2} \delta_{x} U^{n}, \\
\tilde{U}_{\text {midpoint }}^{n+\frac{1}{2}} & =-\frac{1}{2} \delta_{x} \Phi^{n}+\mu_{x} U^{n} .
\end{aligned}
$$

The stencil matrices of the FVEG scheme with trapezoidal and Simpson quadratures for the cell interface integration are given in the Appendix. 
Analogously to the Section 6, we can show that the amplification matrix $\mathcal{T}$ of the first-order FVEG scheme with exact edge integrals is similar to the matrix

$$
\mathcal{Q}=\mathcal{I}-\nu(\mathcal{D}+\mathcal{C})+\nu^{2} \tilde{\mathcal{C}},
$$

where the matrix $\mathcal{D}$ is defined, as before with,

$$
d=2 s_{\xi}^{2}\left(1-\frac{2 \nu}{\pi} s_{\eta}^{2}\right), \quad f=2 s_{\eta}^{2}\left(1-\frac{2 \nu}{\pi} s_{\xi}^{2}\right) .
$$

The matrices $\mathcal{C}$ and $\tilde{\mathcal{C}}$ are given as

$$
\mathcal{C}=\left(\begin{array}{lll}
0 & C_{\xi}-\frac{\nu}{3 \pi} S_{\xi} s_{\eta}^{2} & C_{\eta}-\frac{\nu}{3 \pi} S_{\eta} s_{\xi}^{2} \\
-C_{\xi} & 0 & 0 \\
-C_{\eta} & 0 & 0
\end{array}\right), \quad \tilde{\mathcal{C}}=\left(\begin{array}{lll}
0 & 0 & 0 \\
0 & 0 & C_{\xi \eta} \\
0 & C_{\xi \eta} & 0
\end{array}\right),
$$

where

$$
\begin{aligned}
& C_{\xi}=S_{\xi}\left(1-\frac{2 \nu}{\pi} s_{\eta}^{2}\right), \quad C_{\eta}=S_{\eta}\left(1-\frac{2 \nu}{\pi} s_{\xi}^{2}\right), \\
& C_{\xi \eta}=\frac{-1}{\pi} S_{\xi} S_{\eta} .
\end{aligned}
$$

Since the matrix $\mathcal{C}$ is not skew symmetric, it is now not possible to carry out an analysis similar to the one in Section 6 in order to estimate the stability limit. Instead we use the MATLAB procedure eig to estimate the stability limit. The results are given in Table 2. In Column 2 we present the stability limit of the first-order FVEG scheme with exact edge integrals. The stability limit of this scheme is improved considerably: the scheme is stable approximately up to $\mathrm{CFL}=0.89$. Column 3 demonstrates that the first-order scheme based on the trapezoidal rule is stable up to the natural stability limit 1 . Column 4 shows that the stability of the first-order scheme based on Simpson's rule is also increased: the scheme is stable approximately up to the $\mathrm{CFL}=0.75$.

\begin{tabular}{|c|c|c|c|}
\hline$\frac{c \Delta t}{h}$ & Exact & Trapezoidal & Simpson \\
\hline \hline 0.70 & 1.0000000000 & 1.0000000000 & 1.0000000000 \\
\hline 0.75 & 1.0000000000 & 1.0000000000 & 1.0000000000 \\
\hline 0.76 & 1.0000000000 & 1.0000000000 & 1.0266666667 \\
\hline 0.80 & 1.0000000000 & 1.0000000000 & \\
\hline 0.89 & 1.0000000000 & 1.0000000000 & \\
\hline 0.90 & 1.0007993640 & 1.0000000000 & \\
\hline 1.00 & & 1.0000000000 & \\
\hline 1.01 & & 1.0200000000 & \\
\hline
\end{tabular}

Table 2: Stability limit using $\rho_{\xi, \eta}(\mathcal{T}(\xi, \eta))$

In Figure 4 we plot, using different scales, the eigenvalues of the amplification matrices corresponding to the first order FVEG schemes based on the operator (7.1) - (7.3). Pictures on the top are obtained using exact integration along cell interfaces; in the middle the trapezoidal rule was used to approximate interface integrals. In the bottom part we have plotted eigenvalues of the amplification matrix of the FVEG3 scheme with Simpson's quadrature for the cell interface integrals. Analogously to the previous section, it is possible to include all eigenvalues into the unit disc. 

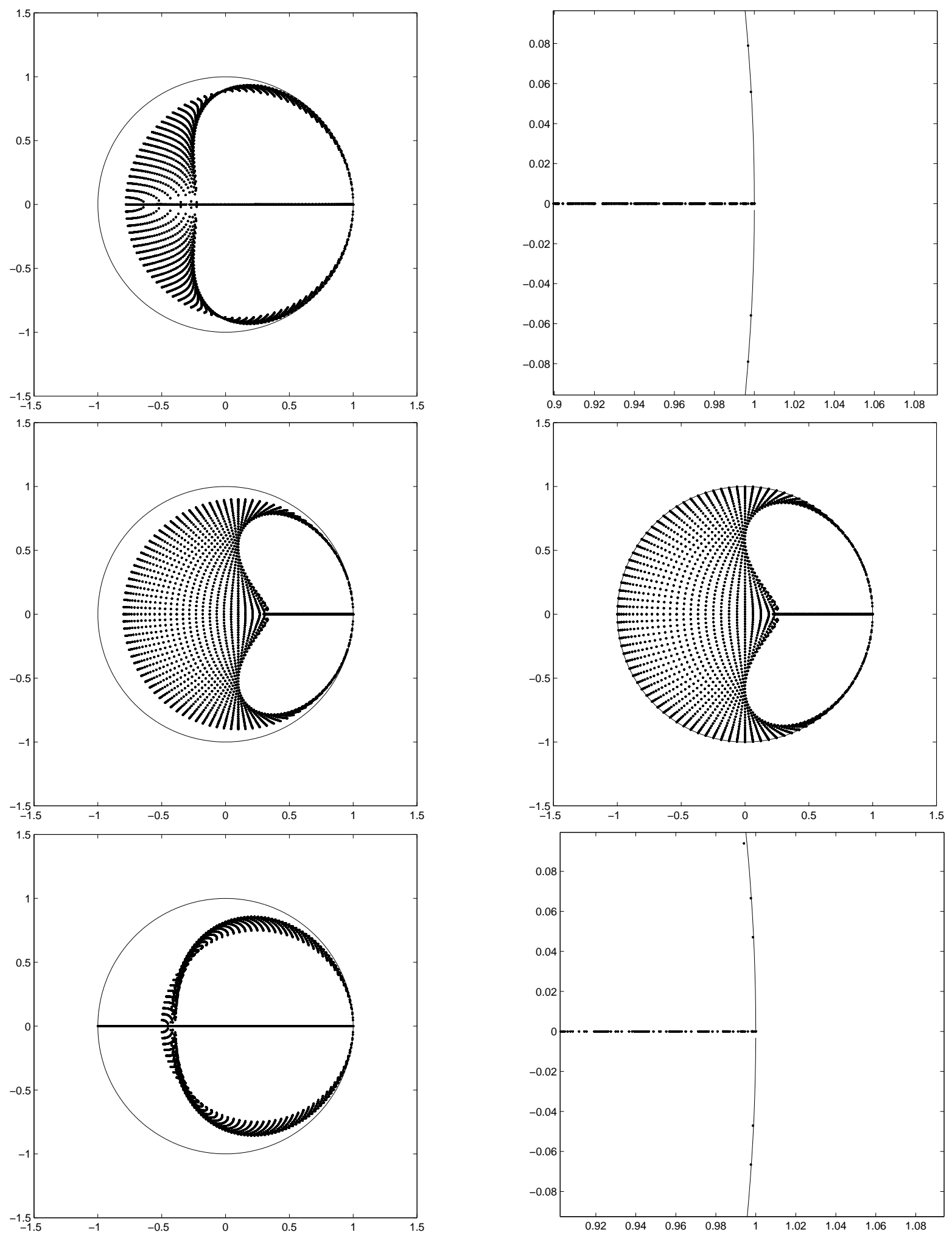

Figure 4: Eigenvalues of the amplification matrices; top: exact interface integration for the $\mathrm{CFL}=0.89$ (right zoom); middle: interface integrals approximated using the trapezoidal rule for the $\mathrm{CFL}=0.9,1.0$., bottom interface integrals approximated by Simpson's rule for the $\mathrm{CFL}=0.75$ (right zoom). 


\section{Approximate evolution operator $E_{\Delta}^{\text {bilin }}$ for piecewise bilinear data}

In this section we investigate the stability of the second-order finite volume schemes proposed by Lukáčová et al. in [11]. These schemes are based on the approximate evolution operator $E_{\Delta}^{\text {bilin }}$, which is given as follows:

$$
\begin{gathered}
\phi_{P}=\left(1-\frac{\pi}{2}\right) \phi_{P}^{\prime}+\frac{1}{2 \pi} \int_{0}^{2 \pi}\left(\frac{\pi}{2} \phi_{Q}-2 u_{Q} \cos \theta-2 v_{Q} \sin \theta\right) \mathrm{d} \theta+O\left(\Delta t^{2}\right), \\
u_{P}=\left(1-\frac{\pi}{4}\right) u_{P}^{\prime}+\frac{1}{2 \pi} \int_{0}^{2 \pi}\left(-2 \cos \theta \phi_{Q}+\frac{\pi}{2} u_{Q}\left(3 \cos ^{2} \theta-1\right)+\frac{3 \pi}{2} v_{Q} \sin \theta \cos \theta\right) \mathrm{d} \theta \\
v_{P}=\left(1-\frac{\pi}{4}\right) v_{P}^{\prime}+\frac{1}{2 \pi} \int_{0}^{2 \pi}\left(-2 \sin \theta \phi_{Q}+\frac{3 \pi}{2} u_{Q} \sin \theta \cos \theta+\frac{\pi}{2} v_{Q}\left(3 \sin ^{2} \theta-1\right)\right) \mathrm{d} \theta \\
+O\left(\Delta t^{2}\right) .
\end{gathered}
$$

Analogously to $E_{\Delta}^{\text {const }}$, this approximate evolution operator is designed so that it computes any one-dimensional linear plane wave propagating in the $x$ - or $y$ - direction exactly; for more details see [11]. In order to obtain second-order finite volume schemes we carry out a recovery stage before applying the approximate evolution operator, see Definition 4.6. The following two types of bilinear recoveries have been considered in [11]

$$
\begin{aligned}
\left.R_{h}^{C} \mathbf{U}\right|_{\Omega_{k l}} & =\left(\mu_{x}^{2} \mu_{y}^{2}+\frac{x-x_{k}}{h} \mu_{x} \mu_{y}^{2} \delta_{x}+\frac{y-y_{l}}{h} \mu_{x}^{2} \mu_{y} \delta_{y}+\frac{\left(x-x_{k}\right)\left(y-y_{l}\right)}{h^{2}} \mu_{x} \mu_{y} \delta_{x} \delta_{y}\right) \mathbf{U}_{k l}, \\
\left.R_{h}^{D} \mathbf{U}\right|_{\Omega_{k l}} & =\left(1+\frac{x-x_{k}}{h} \mu_{x} \mu_{y}^{2} \delta_{x}+\frac{y-y_{l}}{h} \mu_{x}^{2} \mu_{y} \delta_{y}+\frac{\left(x-x_{k}\right)\left(y-y_{l}\right)}{h^{2}} \mu_{x} \mu_{y} \delta_{x} \delta_{y}\right) \mathbf{U}_{k l} .
\end{aligned}
$$

Note, that the recovery (8.4) is continuous while the recovery (8.5) is discontinuous and conservative. We use the midpoint rule to approximate the time integral in the equation (4.7). Denoting the cell interface intermediate value that is computed in the predictor step (4.8) by $\tilde{\mathbf{U}}^{n+\frac{1}{2}}$, we obtain the following schemes

$$
\begin{array}{ll}
\text { scheme A } & \tilde{\mathbf{U}}^{n+\frac{1}{2}}=E_{\Delta}^{\text {bilin }} R_{h}^{C} \mathbf{U}^{n}+E_{\Delta}^{\text {const }}\left(1-\mu_{x}^{2} \mu_{y}^{2}\right) \mathbf{U}^{n}, \\
\text { scheme B } & \tilde{\mathbf{U}}^{n+\frac{1}{2}}=E_{\Delta}^{\text {bilin }} R_{h}^{C} \mathbf{U}^{n}, \\
\text { scheme C } & \tilde{\mathbf{U}}^{n+\frac{1}{2}}=E_{\Delta}^{\text {bilin }} R_{h}^{D} \mathbf{U}^{n} .
\end{array}
$$

Each of these schemes has further two types according to the evaluation of the cell interface integrals. We used the subscripts 1, 2 to distinguish between them. Thus, 1 corresponds to Simpson's rule and 2 to the trapezoidal rule. For example, for the scheme $\mathrm{C}_{2}$ the predicted values along the right cell interface are

$$
\begin{aligned}
& \tilde{\Phi}^{n+\frac{1}{2}}=\left[1+\left(\frac{-\pi}{32}+\frac{\nu}{16}\right) \delta_{x}^{2} \mu_{y}^{2}+\left(\frac{-\pi}{32}+\frac{\nu}{16}\right) \delta_{y}^{2} \mu_{x}^{2}+\left(\frac{\pi}{32}-\frac{\nu}{8}+\frac{\nu^{2}}{32}\right) \delta_{x}^{2} \delta_{y}^{2}\right] \mu_{x} \mu_{y} \Phi^{n} \\
& +\left[\frac{-2}{\pi}+\left(\frac{1}{2 \pi}-\frac{\nu}{8}\right) \mu_{x}^{2} \mu_{y}^{2}+\left(\frac{1}{8 \pi}-\frac{\nu}{16 \pi}\right) \delta_{y}^{2} \mu_{x}^{2}+\left(\frac{-1}{2 \pi}+\frac{\nu}{8}+\frac{\nu}{4 \pi}-\frac{\nu^{2}}{6 \pi}\right) \mu_{x}^{2} \delta_{y}^{2}\right] \delta_{x} \mu_{y} U^{n} \\
& \quad+\left[\frac{-2}{\pi}+\left(\frac{1}{8 \pi}-\frac{\nu}{16 \pi}\right) \delta_{x}^{2} \mu_{y}^{2}+\left(\frac{1}{2 \pi}-\frac{\nu}{8}\right) \mu_{x}^{2} \mu_{y}^{2}+\left(\frac{-1}{2 \pi}+\frac{\nu}{8}+\frac{\nu}{4 \pi}-\frac{\nu^{2}}{6 \pi}\right) \mu_{y}^{2} \delta_{x}^{2}\right] \delta_{y} \mu_{x} V^{n}
\end{aligned}
$$




$$
\begin{aligned}
& \tilde{U}^{n+\frac{1}{2}}=\left[\frac{-2}{\pi}+\left(\frac{1}{2 \pi}-\frac{\nu}{8}\right) \mu_{x}^{2} \mu_{y}^{2}+\left(\frac{1}{8 \pi}-\frac{\nu}{16 \pi}\right) \delta_{y}^{2} \mu_{x}^{2}+\right. \\
& \left.\quad\left(\frac{-1}{2 \pi}+\frac{\nu}{8}+\frac{\nu}{4 \pi}-\frac{\nu^{2}}{6 \pi}\right) \mu_{x}^{2} \delta_{y}^{2}\right] \delta_{x} \mu_{y} \Phi^{n} \\
& +\left[1+\left(\frac{-\pi}{64}+\frac{\nu}{16}\right) \delta_{x}^{2} \mu_{y}^{2}-\frac{\pi}{64} \delta_{y}^{2} \mu_{x}^{2}+\left(\frac{\pi}{64}-\frac{\nu}{16}+\frac{\nu^{2}}{64}\right) \delta_{x}^{2} \delta_{y}^{2}\right] \mu_{x} \mu_{y} U^{n} \\
& +\left[\frac{1}{8}+\left(\frac{1}{16}-\frac{\nu}{8}+\frac{\pi \nu^{2}}{64}\right) \mu_{x}^{2} \mu_{y}^{2}\right] 3 \delta_{x} \delta_{y} V^{n},
\end{aligned}
$$

with the equation for $\tilde{V}^{n+\frac{1}{2}}$ that is analogous to that for $\tilde{U}^{n+\frac{1}{2}}$. Further, we can express analogously the predicted values for the other cell interfaces as well as for other schemes. Substituting the predicted values in the corrector step (4.7) yields, for all second-order finite volume schemes FVEG-A, B, C,

$$
\mathbf{U}_{k l}^{n+1}=\mathbf{U}_{k l}^{n}+\sum_{r=-1}^{1} \sum_{s=-1}^{1} \mathcal{C}_{r s} \mathbf{U}_{k+r l+s}^{n}+\mathcal{C}_{r s}^{x} \mathbf{U}_{x_{k+r l+s}}^{n}+\mathcal{C}_{r s}^{y} \mathbf{U}_{y_{k+r l+s}}^{n}+\mathcal{C}_{r s}^{x y} \mathbf{U}_{x y_{k+r l+s}}^{n},
$$

where $\mathcal{C}_{r s}^{x}, \mathcal{C}_{r s}^{y}$ and $\mathcal{C}_{r s}^{x y}$ are the coefficient matrices corresponding to the approximation of $x-$, $y-$, and $x y$ - slopes. Moreover,

$$
\mathbf{U}_{x_{k+r l+s}}^{n}=\mu_{x} \mu_{y}^{2} \delta_{x} \mathbf{U}_{k+r l+s}^{n}, \mathbf{U}_{y_{k+r l+s}}^{n}=\mu_{x}^{2} \mu_{y} \delta_{y} \mathbf{U}_{k+r l+s}^{n}, \mathbf{U}_{x y_{k+r l+s}}^{n}=\mu_{x} \mu_{y} \delta_{x} \delta_{y} \mathbf{U}_{k+r l+s}^{n} .
$$

Applying a von Neumann analysis and the Fourier transforms we obtain the amplification matrices $\mathcal{T}$. It should be pointed out that their structure is too complicated in order to apply estimates of the spectral radius similar to those in Section 6 for the first order EG3 scheme. Anyway, we can use the standard MATLAB procedure to determine the eigenvalues of $\mathcal{T}$. The corresponding stability limits for the FVEG schemes are given in Table 3.

\begin{tabular}{|c|c|c|}
\hline & Trapezoidal rule & Simpson's rule \\
\hline scheme A & 0.94 & 0.75 \\
scheme B & 0.78 & - \\
scheme C & 0.78 & 0.58 \\
\hline
\end{tabular}

Table 3: Stability limits of the second order FVEG schemes.

We should note that all CFL limits given in the Table 3 have also been confirmed by various numerical experiments. In Figures 5 and 6 we plot, using different scales, the eigenvalues of the amplification matrices corresponding to the second order FVEG schemes; scheme $\mathrm{A}_{i}, \mathrm{~B}_{i}$ and $\mathrm{C}_{i}$, where $i=1,2$. Similarly to the previous cases, these plots indicate that all eigenvalues are included in the unit disc. Note that different quadrature rules have a considerable effect on the form of the spectrum of the resulting amplification matrix. For example, Simpson's quadrature rule for cell interface integrals yields the spectrum which is more compact. Further, it follows from Figure 5 that the second-order FVEG scheme based on the operator (8.1) (8.3) with the continuous non-conservative recovery (8.4) with Simpson's rule, i.e. scheme $\mathrm{B}_{1}$, is unconditionally unstable. This fact has also been confirmed by other numerical tests for the 
wave equation system with discontinuous solution. We have found for all CFL numbers, no matter how small they were chosen, instabilities in the solution for fine enough meshes. We would like to remark that the loss of stability of evolution Galerkin schemes under numerical integration has been also observed even for scalar linear hyperbolic equation by Morton, Priestly and Süli [21].

Remarks: The derivation of the EG and FVEG schemes might be considered at a first sight as a rather complex task. In fact, the finite difference formulation (4.9), (4.10) which uses the stencil matrices, cf. Appendix, is used only for theoretical analysis. In practice we implement the approximate evolution operators (3.7)-(3.9), (7.1)-(7.3) or (8.1)-(8.3) directly. Thus, for example in the FVEG scheme the flux integrals along cell interfaces are approximated by the Simpson or the trapezoidal rule and the only complexity lies in the implementation of the exact integrals of the type $\int_{\alpha}^{\beta} \cos ^{n} \theta \sin ^{m} \theta \mathrm{d} \theta$, where $n, m \geq 0$ - integers, and $\alpha, \beta \in[0,2 \pi]$ are corresponding angles according to a position of the (slanted) Mach cone. Alternatively, the integrals along the sonic circle, i.e. for $\theta$ from 0 to $2 \pi$ can be approximated by a suitable numerical quadrature, which further simplifies the implementation of the FVEG schemes.

Based on our knowledge of the EG and FVEG schemes for regular rectangular two-dimensional meshes some further generalizations have been done. In particular, the FVEG schemes with the approximate evolution operators (3.7)-(3.9), (7.1)-(7.3) as well as (8.1)-(8.3) have been generalized to three-dimensional problems using regular cubic meshes, see [19]. Further, in [26], [27] the FVEG schemes have been generalized for unstructured triangular meshes. Of course, the stability analysis of the EG schemes on general unstructured meshes is much more involved. A possible way would be to apply the energy analysis in the $L^{2}\left(\mathbb{R}^{2}\right)$ norm, i.e. to show at least the weak $L^{2}$-stability $\left\|P_{h} E_{\Delta t}\right\|_{L^{2}\left(\mathbf{R}^{2}\right) \rightarrow L^{2}\left(\mathbf{R}^{2}\right)} \leq 1+C \Delta t$. This is a nontrivial problem, which can be considered in future.

The absorbing boundary conditions have been studied for the EG schemes extensively in [18]. We have considered simple extrapolation boundary conditions, the characteristic boundary conditions as well as perfectly matched layer approach. Numerical experiments reported in [18] indicate that the best results are obtained by combining the EG and FVEG schemes with the perfectly matched layer technique. We have observed no influence of these boundary conditions on stability of the EG schemes. It would be interesting to investigate this question theoretically in future.

\section{Conclusion}

In this paper we have studied the stability of various EG schemes by a von Neumann analysis. The schemes are applied to the linear wave equation system. First, we have discussed theoretically stability estimates of the EG3 schemes, the most favorable one among the finite difference EG schemes considered. Due to the complex structure of the amplification matrix we were able to give theoretical stability estimates only for a simplified problem. Further, we analyzed experimentally the spectral radius of amplification matrix of the EG3 scheme. The experimental analysis indicates that the scheme is stable up to the CFL number 0.58. The stability of the finite volume EG schemes was studied experimentally, too. It has been shown that new quadratures in time for time integrals in the exact evolution operator, which were proposed in our recent paper [11], improve the stability limits considerably. For example, if 

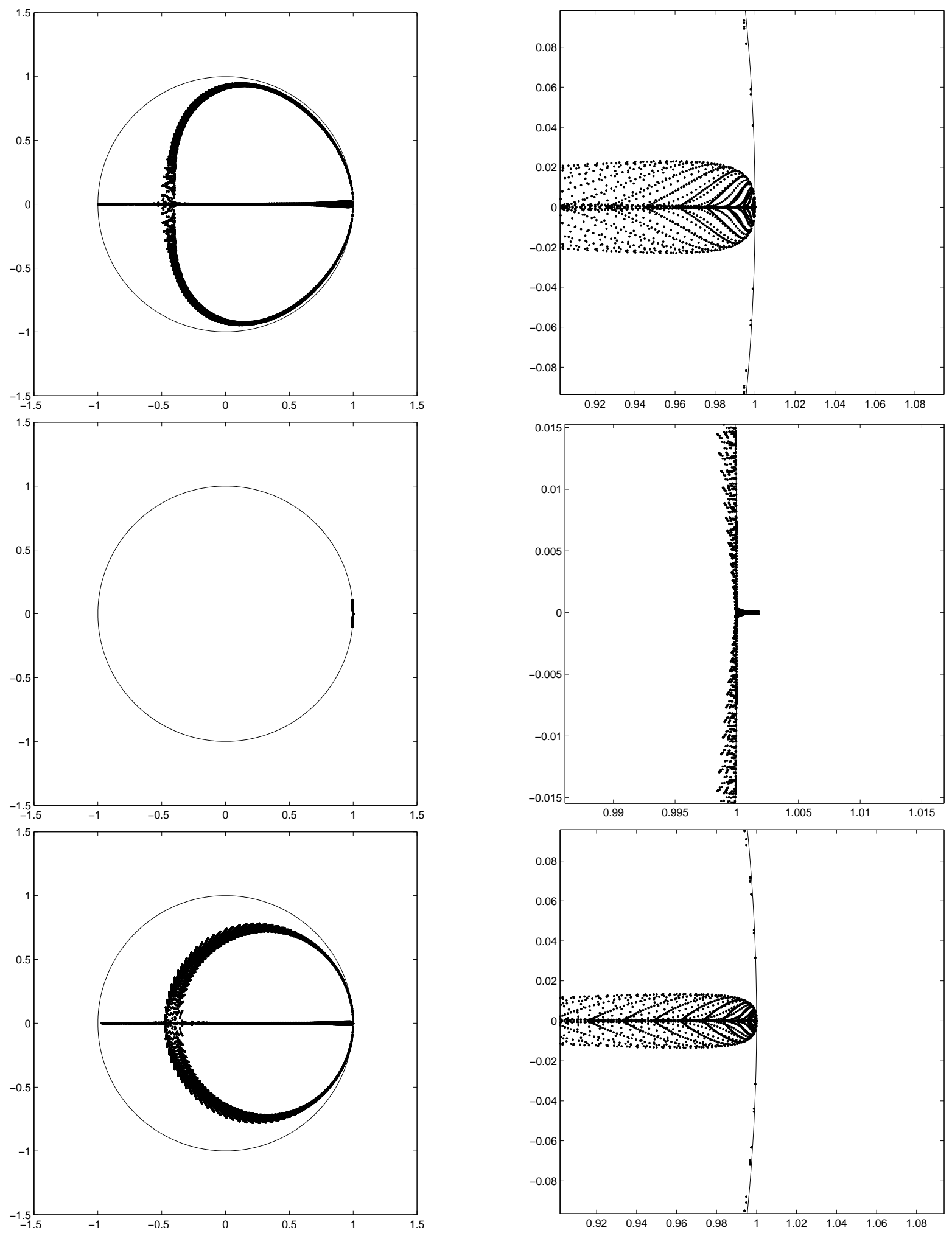

Figure 5: Eigenvalues corresponding to the amplification matrices of the scheme $A_{1}$ $(\mathrm{CFL}=0.75)$, scheme $\mathrm{B}_{1}(\mathrm{CFL}=0.1)$, and the scheme $\mathrm{C}_{1}(\mathrm{CFL}=0.58)$. 

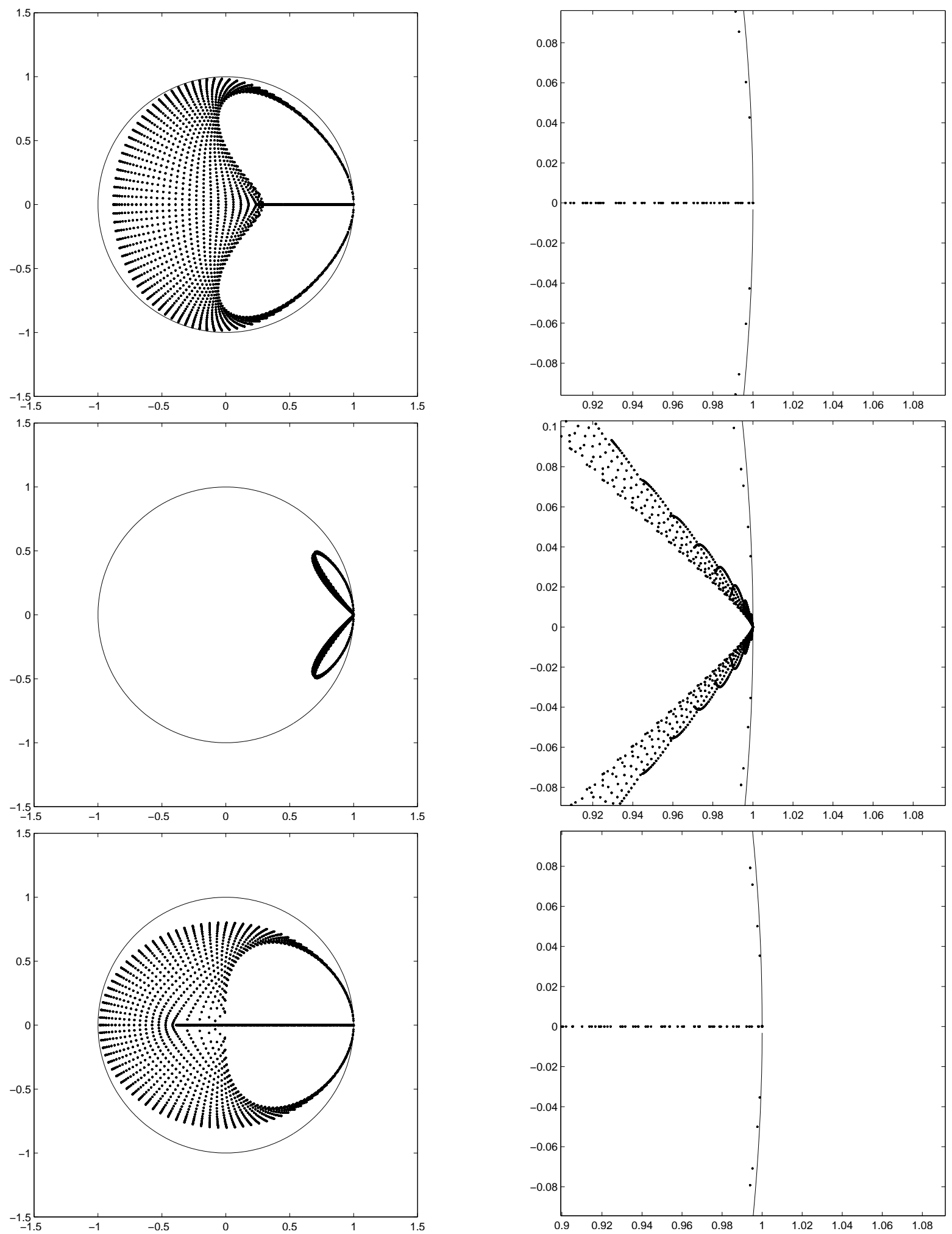

Figure 6: Eigenvalues corresponding to the amplification matrices of the scheme $\mathrm{A}_{2}$ $(\mathrm{CFL}=0.94)$, scheme $\mathrm{B}_{2}(\mathrm{CFL}=0.78)$, scheme $\mathrm{C}_{2}(\mathrm{CFL}=0.78)$. 
the trapezoidal rule is used for the cell interface integrals the CFL number is 1 and 0.94 for the first- and the second-order FVEG schemes, respectively. On the other hand, Simpson's quadrature rule reduces the stability range slightly.

\section{Appendix}

\section{EG3 scheme:}

For the discrete form of the scheme see (4.5), (4.9), (4.10), and (3.7)-(3.9) for the approximate evolution operator EG3

$$
\begin{aligned}
& \alpha^{1}:=\left\{\begin{array}{rrr}
\frac{\nu^{2}}{4 \pi} & \frac{\nu}{\pi}-\frac{\nu^{2}}{2 \pi} & \frac{\nu^{2}}{4 \pi} \\
\frac{\nu}{\pi}-\frac{\nu^{2}}{2 \pi} & -\frac{4 \nu}{\pi}+\frac{\nu^{2}}{\pi} & \frac{\nu}{\pi}-\frac{\nu^{2}}{2 \pi} \\
\frac{\nu^{2}}{4 \pi} & \frac{\nu}{\pi}-\frac{\nu^{2}}{2 \pi} & \frac{\nu^{2}}{4 \pi}
\end{array}\right\}, \quad \beta^{1}:=\left\{\begin{array}{rrr}
\frac{\nu^{2}}{3 \pi} & 0 & \frac{-\nu^{2}}{3 \pi} \\
\frac{\nu}{2}-\frac{2 \nu^{2}}{3 \pi} & 0 & -\frac{\nu}{2}+\frac{2 \nu^{2}}{3 \pi} \\
\frac{\nu^{2}}{3 \pi} & 0 & -\frac{\nu^{2}}{3 \pi}
\end{array}\right\} \\
& \gamma^{1}:=\left\{\begin{array}{rrr}
-\frac{\nu^{2}}{3 \pi} & -\frac{\nu}{2}+\frac{2 \nu^{2}}{3 \pi} & -\frac{\nu^{2}}{3 \pi} \\
0 & 0 & 0 \\
\frac{\nu^{2}}{3 \pi} & \frac{\nu}{2}-\frac{2 \nu^{2}}{3 \pi} & \frac{\nu^{2}}{3 \pi}
\end{array}\right\}, \alpha^{2}:=\left\{\begin{array}{rrr}
\frac{\nu^{2}}{3 \pi} & 0 & -\frac{\nu^{2}}{3 \pi} \\
\frac{\nu}{2}-\frac{2 \nu^{2}}{3 \pi} & 0 & -\frac{\nu}{2}+\frac{2 \nu^{2}}{3 \pi} \\
\frac{\nu^{2}}{3 \pi} & 0 & \frac{-\nu^{2}}{3 \pi}
\end{array}\right\} \\
& \beta^{2}:=\left\{\begin{array}{rrr}
\frac{\nu^{2}}{8 \pi} & -\frac{\nu^{2}}{4 \pi} & \frac{\nu^{2}}{8 \pi} \\
\frac{\nu}{\pi}-\frac{\nu^{2}}{4 \pi} & -\frac{2 \nu}{\pi}+\frac{\nu^{2}}{2 \pi} & \frac{\nu}{\pi}-\frac{\nu^{2}}{4 \pi} \\
\frac{\nu^{2}}{8 \pi} & -\frac{\nu^{2}}{4 \pi} & \frac{\nu^{2}}{8 \pi}
\end{array}\right\}, \gamma^{2}:=\left\{\begin{array}{rrr}
-\frac{3 \nu^{2}}{32} & 0 & \frac{3 \nu^{2}}{32} \\
0 & 0 & 0 \\
\frac{3 \nu^{2}}{32} & 0 & -\frac{3 \nu^{2}}{32}
\end{array}\right\} \\
& \alpha^{3}:=\left\{\begin{array}{rrr}
-\frac{\nu^{2}}{3 \pi} & -\frac{\nu}{2}+\frac{2 \nu^{2}}{3 \pi} & -\frac{\nu^{2}}{3 \pi} \\
0 & 0 & 0 \\
\frac{\nu^{2}}{3 \pi} & +\frac{\nu}{2}-\frac{2 \nu^{2}}{3 \pi} & \frac{\nu^{2}}{3 \pi}
\end{array}\right\}, \beta^{3}:=\left\{\begin{array}{rrr}
-\frac{3 \nu^{2}}{32} & 0 & \frac{3 \nu^{2}}{32} \\
0 & 0 & 0 \\
\frac{3 \nu^{2}}{32} & 0 & -\frac{3 \nu^{2}}{32}
\end{array}\right\}, \gamma^{3}:=\left\{\begin{array}{rrr}
\frac{\nu^{2}}{8 \pi} & \frac{\nu}{\pi}-\frac{\nu^{2}}{4 \pi} & \frac{\nu^{2}}{8 \pi} \\
-\frac{\nu^{2}}{4 \pi} & -\frac{2 \nu}{\pi}+\frac{\nu^{2}}{2 \pi} & -\frac{\nu^{2}}{4 \pi} \\
\frac{\nu^{2}}{8 \pi} & \frac{\nu}{\pi}-\frac{\nu^{2}}{4 \pi} & \frac{\nu^{2}}{8 \pi}
\end{array}\right\} .
\end{aligned}
$$


FVEG scheme with $E_{\Delta}^{\text {const }}$ operator using exact cell inteface integration:

For the discrete form of the scheme see (4.7)-(4.10), and (7.1)-(7.3) for the approximate evolution operator $E_{\Delta}^{\text {const }}$

$$
\begin{aligned}
& \alpha^{1}:=\left\{\begin{array}{rrr}
\frac{\nu^{2}}{2 \pi} & \frac{\nu}{2}-\frac{\nu^{2}}{\pi} & \frac{\nu^{2}}{2 \pi} \\
\frac{\nu}{2}-\frac{\nu^{2}}{\pi} & -2 \nu+\frac{2 \nu^{2}}{\pi} & \frac{\nu}{2}-\frac{\nu^{2}}{\pi} \\
\frac{\nu^{2}}{2 \pi} & \frac{\nu}{2}-\frac{\nu^{2}}{\pi} & \frac{\nu^{2}}{2 \pi}
\end{array}\right\}, \quad \beta^{1}:=\left\{\begin{array}{rrr}
\frac{7 \nu^{2}}{24 \pi} & 0 & \frac{-7 \nu^{2}}{24 \pi} \\
\frac{\nu}{2}-\frac{7 \nu^{2}}{12 \pi} & 0 & \frac{-\nu}{2}+\frac{7 \nu^{2}}{12 \pi} \\
\frac{7 \nu^{2}}{24 \pi} & 0 & \frac{-7 \nu^{2}}{24 \pi}
\end{array}\right\}, \\
& \gamma^{1}:=\left\{\begin{array}{rrr}
\frac{-7 \nu^{2}}{24 \pi} & \frac{-\nu}{2}+\frac{7 \nu^{2}}{12 \pi} & \frac{-7 \nu^{2}}{24 \pi} \\
0 & 0 & 0 \\
\frac{7 \nu^{2}}{24 \pi} & \frac{\nu}{2}-\frac{7 \nu^{2}}{12 \pi} & \frac{7 \nu^{2}}{24 \pi}
\end{array}\right\}, \alpha^{2}:=\left\{\begin{array}{rrr}
\frac{\nu^{2}}{4 \pi} & 0 & \frac{-\nu^{2}}{4 \pi} \\
\frac{\nu}{2}-\frac{\nu^{2}}{2 \pi} & 0 & \frac{-\nu}{2}+\frac{\nu^{2}}{2 \pi} \\
\frac{\nu^{2}}{4 \pi} & 0 & \frac{-\nu^{2}}{4 \pi}
\end{array}\right\}, \\
& \beta^{2}:=\left\{\begin{array}{lcr}
\frac{\nu^{2}}{4 \pi} & -\frac{\nu^{2}}{2 \pi} & \frac{\nu^{2}}{4 \pi} \\
\frac{\nu}{2}-\frac{\nu^{2}}{2 \pi} & -\nu+\frac{\nu^{2}}{\pi} & \frac{\nu}{2}-\frac{\nu^{2}}{2 \pi} \\
\frac{\nu^{2}}{4 \pi} & -\frac{\nu^{2}}{2 \pi} & \frac{\nu^{2}}{4 \pi}
\end{array}\right\}, \gamma^{2}:=\left\{\begin{array}{rrr}
\frac{-\nu^{2}}{4 \pi} & 0 & \frac{\nu^{2}}{4 \pi} \\
0 & 0 & 0 \\
\frac{\nu^{2}}{4 \pi} & 0 & \frac{-\nu^{2}}{4 \pi}
\end{array}\right\} \\
& \alpha^{3}:=\left\{\begin{array}{rrc}
\frac{-\nu^{2}}{4 \pi} & \frac{-\nu}{2}+\frac{\nu^{2}}{2 \pi} & \frac{-\nu^{2}}{4 \pi} \\
0 & 0 & 0 \\
\frac{\nu^{2}}{4 \pi} & \frac{\nu}{2}-\frac{\nu^{2}}{2 \pi} & \frac{\nu^{2}}{4 \pi}
\end{array}\right\}, \beta^{3}:=\left\{\begin{array}{rrr}
\frac{-\nu^{2}}{4 \pi} & 0 & \frac{\nu^{2}}{4 \pi} \\
0 & 0 & 0 \\
\frac{\nu^{2}}{4 \pi} & 0 & \frac{-\nu^{2}}{4 \pi}
\end{array}\right\}, \\
& \gamma^{3}:=\left\{\begin{array}{ccr}
\frac{\nu^{2}}{4 \pi} & \frac{\nu}{2}-\frac{\nu^{2}}{2 \pi} & \frac{\nu^{2}}{4 \pi} \\
-\frac{\nu^{2}}{2 \pi} & -\nu+\frac{\nu^{2}}{\pi} & -\frac{\nu^{2}}{2 \pi} \\
\frac{\nu^{2}}{4 \pi} & \frac{\nu}{2}-\frac{\nu^{2}}{2 \pi} & \frac{\nu^{2}}{4 \pi}
\end{array}\right\}
\end{aligned}
$$


FVEG with $E_{\Delta}^{\text {const }}$ operator using Simpson's quadrature for cell interface integration:

For the discrete form of the scheme see (4.7),(4.8) with Simpson's quadrature, (4.9), (4.10), and (7.1)-(7.3) for the approximate evolution operator $E_{\Delta}^{\text {const }}$

$$
\begin{aligned}
& \alpha^{1}:=\left\{\begin{array}{rrr}
\frac{\nu}{12} & \frac{\nu}{3} & \frac{\nu}{12} \\
\frac{\nu}{3} & -\frac{5 \nu}{3} & \frac{\nu}{3} \\
\frac{\nu}{12} & \frac{\nu}{3} & \frac{\nu}{12}
\end{array}\right\}, \beta^{1}:=\left\{\begin{array}{rrr}
\frac{\nu}{24}\left(1+\frac{1}{\pi}\right) & 0 & -\frac{\nu}{24}\left(1+\frac{1}{\pi}\right) \\
\frac{\nu}{24}\left(10-\frac{2}{\pi}\right) & 0 & -\frac{\nu}{24}\left(10-\frac{2}{\pi}\right) \\
\frac{\nu}{24}\left(1+\frac{1}{\pi}\right) & 0 & -\frac{\nu}{24}\left(1+\frac{1}{\pi}\right)
\end{array}\right\}, \\
& \gamma^{1}:=\left\{\begin{array}{rrr}
-\frac{\nu}{24}\left(1+\frac{1}{\pi}\right) & -\frac{\nu}{24}\left(10-\frac{2}{\pi}\right) & -\frac{\nu}{24}\left(1+\frac{1}{\pi}\right) \\
0 & 0 & 0 \\
\frac{\nu}{24}\left(1+\frac{1}{\pi}\right) & \frac{\nu}{24}\left(10-\frac{2}{\pi}\right) & \frac{\nu}{24}\left(1+\frac{1}{\pi}\right)
\end{array}\right\}, \alpha^{2}:=\left\{\begin{array}{rrr}
\frac{\nu}{24} & 0 & -\frac{\nu}{24} \\
\frac{10 \nu}{24} & 0 & -\frac{10 \nu}{24} \\
\frac{\nu}{24} & 0 & -\frac{\nu}{24}
\end{array}\right\}, \\
& \beta^{2}:=\left\{\begin{array}{rrr}
\frac{\nu}{24} & -\frac{2 \nu}{24} & \frac{\nu}{24} \\
\frac{10 \nu}{24} & -\frac{20 \nu}{24} & \frac{10 \nu}{24} \\
\frac{\nu}{24} & -\frac{2 \nu}{24} & \frac{\nu}{24}
\end{array}\right\}, \quad \gamma^{2}:=\left\{\begin{array}{rrr}
-\frac{\nu}{24} & 0 & \frac{\nu}{24} \\
0 & 0 & 0 \\
\frac{\nu}{24} & 0 & -\frac{\nu}{24}
\end{array}\right\} \\
& \alpha^{3}:=\left\{\begin{array}{rrr}
-\frac{\nu}{24} & -\frac{10 \nu}{24} & -\frac{\nu}{24} \\
0 & 0 & 0 \\
\frac{\nu}{24} & \frac{10 \nu}{24} & \frac{\nu}{24}
\end{array}\right\}, \quad \beta^{3}:=\left\{\begin{array}{rrr}
-\frac{\nu}{24} & 0 & \frac{\nu}{24} \\
0 & 0 & 0 \\
\frac{\nu}{24} & 0 & -\frac{\nu}{24}
\end{array}\right\} \\
& \gamma^{3}:=\left\{\begin{array}{rrr}
\frac{\nu}{24} & \frac{10 \nu}{24} & \frac{\nu}{24} \\
-\frac{2 \nu}{24} & -\frac{20 \nu}{24} & -\frac{2 \nu}{24} \\
\frac{\nu}{24} & \frac{10 \nu}{24} & \frac{\nu}{24}
\end{array}\right\} .
\end{aligned}
$$


FVEG with $E_{\Delta}^{c o n s t}$ operator using the trapezoidal quadrature for cell interface integration:

For the discrete form of the scheme see (4.7), (4.8) with the trapezoidal quadrature, (4.9), (4.10), and (7.1)-(7.3) for the approximate evolution operator $E_{\Delta}^{\text {const }}$

$$
\begin{aligned}
& \alpha^{1}:=\left\{\begin{array}{ccc}
\frac{\nu}{4} & 0 & \frac{\nu}{4} \\
0 & -\nu & 0 \\
\frac{\nu}{4} & 0 & \frac{\nu}{4}
\end{array}\right\}, \beta^{1}:=\left\{\begin{array}{ccc}
\frac{\nu}{8}\left(1+\frac{1}{\pi}\right) & 0 & -\frac{\nu}{8}\left(1+\frac{1}{\pi}\right) \\
\frac{\nu}{8}\left(2-\frac{2}{\pi}\right) & 0 & -\frac{\nu}{8}\left(2-\frac{2}{\pi}\right) \\
\frac{\nu}{8}\left(1+\frac{1}{\pi}\right) & 0 & -\frac{\nu}{8}\left(1+\frac{1}{\pi}\right)
\end{array}\right\}, \\
& \gamma^{1}:=\left\{\begin{array}{rrr}
-\frac{\nu}{8}\left(1+\frac{1}{\pi}\right) & -\frac{\nu}{8}\left(2-\frac{2}{\pi}\right) & -\frac{\nu}{8}\left(1+\frac{1}{\pi}\right) \\
0 & 0 & 0 \\
\frac{\nu}{8}\left(1+\frac{1}{\pi}\right) & \frac{\nu}{8}\left(2-\frac{2}{\pi}\right) & \frac{\nu}{8}\left(1+\frac{1}{\pi}\right)
\end{array}\right\}, \alpha^{2}:=\left\{\begin{array}{rrr}
\frac{\nu}{8} & 0 & -\frac{\nu}{8} \\
\frac{2 \nu}{8} & 0 & -\frac{2 \nu}{8} \\
\frac{\nu}{8} & 0 & -\frac{\nu}{8}
\end{array}\right\}, \\
& \beta^{2}:=\left\{\begin{array}{rrr}
\frac{\nu}{8} & -\frac{2 \nu}{8} & \frac{\nu}{8} \\
\frac{2 \nu}{8} & -\frac{4 \nu}{8} & \frac{2 \nu}{8} \\
\frac{\nu}{8} & -\frac{2 \nu}{8} & \frac{\nu}{8}
\end{array}\right\}, \quad \gamma^{2}:=\left\{\begin{array}{rrr}
-\frac{\nu}{8} & 0 & \frac{\nu}{8} \\
0 & 0 & 0 \\
\frac{\nu}{8} & 0 & -\frac{\nu}{8}
\end{array}\right\}, \\
& \alpha^{3}:=\left\{\begin{array}{rrr}
-\frac{\nu}{8} & -\frac{2 \nu}{8} & -\frac{\nu}{8} \\
0 & 0 & 0 \\
\frac{\nu}{8} & \frac{2 \nu}{8} & \frac{\nu}{8}
\end{array}\right\}, \quad \beta^{3}:=\left\{\begin{array}{rrr}
-\frac{\nu}{8} & 0 & \frac{\nu}{8} \\
0 & 0 & 0 \\
\frac{\nu}{8} & 0 & -\frac{\nu}{8}
\end{array}\right\}, \\
& \gamma^{3}:=\left\{\begin{array}{rrr}
\frac{\nu}{8} & \frac{2 \nu}{8} & \frac{\nu}{8} \\
-\frac{2 \nu}{8} & -\frac{4 \nu}{8} & -\frac{2 \nu}{8} \\
\frac{\nu}{8} & \frac{2 \nu}{8} & \frac{\nu}{8}
\end{array}\right\} .
\end{aligned}
$$




\section{References}

[1] R. Courant and K. O. Friedrichs. Supersonic flow and shock waves. Interscience Publ., Wiley, New York, 1948. [Reprinted by Springer-Verlag, New York, 1985].

[2] S. Depeyre. A stability analysis for finite volume schemes applied to the Maxwell system, $M^{2} A N, 33(3): 443-458,1999$.

[3] M. Fey. Multidimensional upwinding, Part I. The method of transport for solving the Euler equations. J. Comp. Phys., 143:159-180, 1998.

[4] M. Fey. Multidimensional upwinding, Part II. Decomposition of the Euler equations into advection equations. J. Comp. Phys., 143:181-199, 1998.

[5] G. Hämmerlin and K.-H. Hoffmann. Numerical Mathematics, Trans. from the German by Larry Schumaker. (English) Undergraduate Texts in Mathematics; Readings in Mathematics. New York: Springer-Verlag, 1991.

[6] T. Kröger and M. Lukáčová-Medvidová. An evolution Galerkin scheme for the shallow water magnetohydrodynamic equations in two space dimensions. J. Comp. Phys., 206(1):122-149, 2005.

[7] R.J. LeVeque. Wave propagation algorithms for multidimensional hyperbolic systems. J. Comp. Phys., 131:327-353, 1997.

[8] P. Lin, K.W. Morton, and E. Süli. Euler characteristic Galerkin scheme with recovery, $M^{2} A N, 27(7): 863-894,1993$.

[9] P. Lin, K.W. Morton, and E. Süli. Characteristic Galerkin schemes for scalar conservation laws in two and three space dimensions, SIAM J. Numer. Anal., 34(2):779-796, 1997.

[10] M. Lukáčová-Medvidová, K. W. Morton, and G. Warnecke. Finite volume evolution Galerkin methods for Euler equations of gas dynamics. Int. J. Num. Methods in Fluids, 40(3-4):425-434, 2002.

[11] M. Lukáčová-Medvid’ová, K.W. Morton, and G. Warnecke. Finite volume evolution Galerkin (FVEG) methods for hyperbolic systems, SIAM J. Sci. Comp., 26(1):1-30, 2004 .

[12] M. Lukáčová-Medvidová, K.W. Morton, and G. Warnecke. High resolution finite volume evolution Galerkin schemes for multidimensional conservation laws. Proceedings of ENUMATH'99, World Scientific Publishing Company, Singapore, 1999.

[13] M. Lukáčová-Medvidová, K.W. Morton, and G. Warnecke. Evolution Galerkin methods for hyperbolic systems in two space dimensions. Math. Comput., 69(232):1355-1384, 2000 .

[14] M. Lukáčová-Medvidová, K.W. Morton, and G. Warnecke. Evolution Galerkin methods for multidimensional hyperbolic systems. Proceedings of ECCOMAS 2000, Barcelona, $1-14,2000$. 
[15] M. Lukáčová-Medvid’ová, J. Saibertová, and G. Warnecke. Finite volume evolution Galerkin methods for nonlinear hyperbolic systems. J. Comput. Phys., 183:533-562, 2002.

[16] M. Lukáčová-Medvidová, J. Saibertová, G. Warnecke, and Y. Zahaykah. On evolution Galerkin methods for the Maxwell and the linearized Euler equations, Appl. Math., 49(5):415-439, 2004.

[17] M. Lukáčová-Medvid’ová, G. Warnecke, and Y. Zahaykah. Third order finite volume evolution Galerkin (FVEG) methods for two-dimensional wave equation system, J. Numer. Math., 11(3):235-251, 2003.

[18] M. Lukáčová-Medvidová, G. Warnecke, and Y. Zahaykah. On the boundary conditions for EG-methods applied to the two-dimensional wave equation systems, ZAMM, 84(4):237-251, 2004.

[19] M. Lukáčová-Medvidová, G. Warnecke, and Y. Zahaykah. Finite volume evolution Galerkin (FVEG) methods for three-dimensional wave equation system, submitted 2004, pp. 16.

[20] M. Lukáčová-Medvid’ová, S. Noelle, and M. Kraft: Well-balanced finite volume evolution Galerkin methods for the shallow water equations, submitted, 2005, pp. 27.

[21] K.W. Morton, A. Priestly, and E. Süli. Convergence of the Lagrange-Galerkin method with non-exact integration, $M^{2}$ AN Math. Modell. Num. Anal., 22(4):625-653, 1988.

[22] S. Noelle. The MOT-ICE: a new high-resolution wave-propagation algorithm for multidimensional systems of conservative laws based on Fey's method of transport. J. Comp. Phys., 164:283-334, 2000.

[23] S. Ostkamp. Multidimensional characteristic Galerkin schemes and evolution operators for hyperbolic systems. Math. Meth. Appl. Sci., 20:1111-1125, 1997.

[24] R. D. Richtmyer and K. W. Morton. Difference methods for initial-value problems. Interscience Publ., John Wiley \& Sons, New York, London, Sydney, 1967.

[25] P. Roe. Discrete models for the numerical analysis of time-dependent multidimensional gas dynamics. J. Comp. Phys., 63:458-476, 1986.

[26] Qurrat-ul-Ain. Multidimensional Schemes for Hyperbolic Conservation Laws on Triangular Meshes. Dissertation, University of Magdeburg, 2005.

[27] Qurrat-ul-Ain, G. Warnecke, and Y. Zahaykah. On the finite volume evolution Galerkin (FVEG) methods for two-dimensional first order hyperbolic systems on structured and unstructured triangular meshes, in preparation.

[28] Y. Zahaykah. Evolution Galerkin Schemes and Discrete Boundary Conditions for Multidimensional First Order Systems. Dissertation, University of Magdeburg, 2002. 